\title{
Application of single-cell RNA sequencing in optimizing a combinatorial therapeutic strategy in metastatic renal cell carcinoma
}

\author{
Kyu-Tae Kim ${ }^{1 \dagger}$, Hye Won Lee ${ }^{2 \dagger}$, Hae-Ock Lee ${ }^{1,3}$, Hye Jin Song ${ }^{4}$, Da Eun Jeong ${ }^{5}$, Sang Shin ${ }^{5}$, Hyunho Kim ${ }^{6}$, \\ Yoojin Shin ${ }^{6}$, Do-Hyun Nam,7, Byong Chang Jeong ${ }^{8}$, David G. Kirsch ${ }^{9,10}$, Kyeung Min Joo ${ }^{4,5^{*}}$ \\ and Woong-Yang Park ${ }^{1,3,5^{*}}$
}

\begin{abstract}
Background: Intratumoral heterogeneity hampers the success of marker-based anticancer treatment because the targeted therapy may eliminate a specific subpopulation of tumor cells while leaving others unharmed. Accordingly, a rational strategy minimizing survival of the drug-resistant subpopulation is essential to achieve long-term therapeutic efficacy.

Results: Using single-cell RNA sequencing (RNA-seq), we examine the intratumoral heterogeneity of a pair of primary renal cell carcinoma and its lung metastasis. Activation of drug target pathways demonstrates considerable variability between the primary and metastatic sites, as well as among individual cancer cells within each site. Based on the prediction of multiple drug target pathway activation, we derive a combinatorial regimen co-targeting two mutually exclusive pathways for the metastatic cancer cells. This combinatorial strategy shows significant increase in the treatment efficacy over monotherapy in the experimental validation using patient-derived xenograft platforms in vitro and in vivo.

Conclusions: Our findings demonstrate the investigational application of single-cell RNA-seq in the design of an anticancer regimen. The approach may overcome intratumoral heterogeneity which hampers the success of precision medicine.
\end{abstract}

Keywords: Single cell analysis, Renal cell carcinoma, Patient-derived xenograft, Tumor heterogeneity, Drug response

\section{Background}

Clear cell renal cell carcinoma (ccRCC), the most prevalent type of sporadic kidney cancer, is often associated with malignant disease progression and poor therapeutic outcomes [1]. A major underlying genetic alteration in ccRCC is the von Hippel-Lindau ( $V H L)$ tumor-suppressor gene, whose deregulation stimulates an oncologic metabolic shift [2]. Signaling pathways involved in this metabolic shift have been proposed as potential therapeutic targets, including epidermal

\footnotetext{
*Correspondence: kmjoo@skku.edu; woongyang.park@samsung.com ${ }^{\dagger}$ Equal contributors

${ }^{4}$ Departments of Anatomy and Cell Biology, Sungkyunkwan University School of Medicine, Seoul, South Korea

${ }^{1}$ Samsung Genome Institute, Samsung Medical Center, Seoul, South Korea Full list of author information is available at the end of the article
}

growth factor receptor (EGFR) [3], vascular endothelial growth factor (VEGFR) [4], or mammalian target of rapamycin (mTOR) pathways [5]. Although targeting these pathways significantly improved progression-free survival, the outgrowth of drug-resistant clones reduced the clinical efficacy and remains a clinical challenge that must be overcome [1].

Approximately $30 \%$ of patients with renal cell carcinoma (RCC) are diagnosed with metastases [6]. Metastatic renal cell carcinoma (mRCC) evolves from primary RCC (pRCC) and harbors multiple subpopulations with distinct genomic $[7,8]$ and transcriptomic [9-11] features. Such intratumoral heterogeneity (ITH) is augmented by spatiotemporal tumor evolution $[7,8,12]$. The existence of disparate admixtures of cancer cells across $\mathrm{mRCC}$ and 
pRCC typically leads to significant differences in their sensitivity to therapies [13] and rare complete response to targeted molecular agents [1]. Therefore, independent investigation of $\mathrm{mRCC}$ and $\mathrm{pRCC}$ tumor tissues is essential to comprehend the intratumoral landscape within a patient and ultimately to achieve sustainable therapeutic benefit through a rational drug combination design. The magnitude of ITH in ccRCC has been discerned in detail by recent approaches of sequencing multiregional biopsied specimens $[7,8]$ or single cells [14]. However, experimental applications of the analyzed ITH signature to effectively eradicate tumor cells have not been extensively investigated.

Accurate identification of disease-causing sequence variants and driving pathways is essential to minimize drug resistance or tumor relapse with targeted therapeutics [15]. Genomic mutations, however, may have limited functional significance as druggable targets, according to previous findings that drug responses can be widely different even in genetically homogeneous cancer cell lines $[16,17]$. A systematic assessment from the National Cancer Institute and the Dialogue on Reverse Engineering Assessment and Methods (NCI-DREAM) project has shown that gene expression profiling has the best predictive power among independent profiling platforms, with increasing power upon data integration [18]. In the prediction of drug sensitivity in cancer cells, transcriptome profiling can enhance our understanding of how the cellular mechanism is functionally perturbed in response to drug treatment [19]. Moreover, considering that single targeting agents may eliminate a certain subpopulation of tumor cells while leaving others unharmed, it is necessary to analyze the tumor transcriptome at high resolution to detect drug-resistant clones that may be concealed within ITH. Gene expression profiling at a single-cell resolution may enable modeling of functional heterogeneity and identification of subpopulations with specific drug responsive signatures.

Here, we used single-cell RNA sequencing (scRNAseq) not only to elucidate transcriptional heterogeneity during the metastatic progression, but also to design an optimized combination of targeted agents against metastatic RCC. On the basis of single-cell transcriptome analysis, identification of cellular subpopulations with distinct activation status of signaling pathways allowed us to postulate a combinational therapeutic regimen with an increased likelihood of covering all potentially targetable cancer cells.

\section{Results}

Establishment of patient-derived xenografts from paired pRCC and mRCC

The patient exhibited intrinsic refractoriness to sequential conventional therapies, including pazopanib, everolimus, and high-dose interleukin-2, resulting in rapid multiorgan dissemination of cancer cells after pulmonary metastectomy (Fig. 1a). To verify the data-driven prediction of putative therapeutic targets we used paired pRCC and mRCC patient-derived xenografts (PDX) for RCC, which enabled us to understand the ITH at the cellular level [20] with the benefits of recapitulating the molecular, genetic, and histopathologic heterogeneity of the parental tumors [21-23]. The parental tumors and PDXs shared histopathologic characteristics, including subtype-specific morphologic features and differentiation status (Additional file 1: Figure S1A). The cancer cell fractions were highly enriched in the PDX models (Additional file 1: Figure S1B), as previously observed [24]. Although there were discordant somatic single-nucleotide variants (SSNVs) between parental tumors and PDX samples (Additional file 1: Figure S1C) from whole-exome sequencing (WES) analysis, genes that are frequently mutated in ccRCCs [25] persisted in all tumor samples (Additional file 1: Figure S1D). Copy number variations detected from array comparative genomic hybridization (aCGH) analysis in parental tumors were also preserved in PDX tumors (Additional file 1: Figure S1E and F). Together, these data indicate that human tumors from $\mathrm{pRCC}$ and $\mathrm{mRCC}$ were propagated through xenograft engraftments with consistent features of histopathology and genomic landscape.

\section{Evolutionary genomic trajectories during tumor progression and metastasis}

In recognition of that genomic features were consistently propagated with higher cancer cell fraction ( 100\%) through xenograft passaging (Additional file 1: Figure S1), we investigated genomic architectures in the pRCC and mRCC tumors from PDX samples to understand the clonal evolution associated with the spatiotemporal tumor progression. WES analysis of primary and paired metastatic samples revealed that $23.5 \%$ of SSNVs were shared (Additional file 2: Figure S2A). In particular, a $V H L$ D121G mutation was found in both samples with high allele frequencies ( 1.0, Additional file 2: Figure S2A and Additional file 3: Table S1), suggesting that it might be a founder event in tumor evolution $[7,8]$. Variant allele frequencies (VAF) of the shared SSNVs were typically higher than those of SSNVs exclusively observed in mRCC (38\%) or pRCC (38.6\%) (Additional file 2: Figure S2A). Discordant SSNVs in mRCC and pRCC might result from the gradual increase in point mutations and clonal selection with tumor evolution, as previously reported $[7,8]$. In contrast, somatic copy number alterations (SCNAs) in mRCC were similar to those in pRCC (Additional file 2: Figure S2B), with 5q amplifications detected only in pRCC (Additional file 2: Figure S2C). Integrated analyses of WES and aCGH to infer evolutionary trajectories showed that major clones harboring driver 


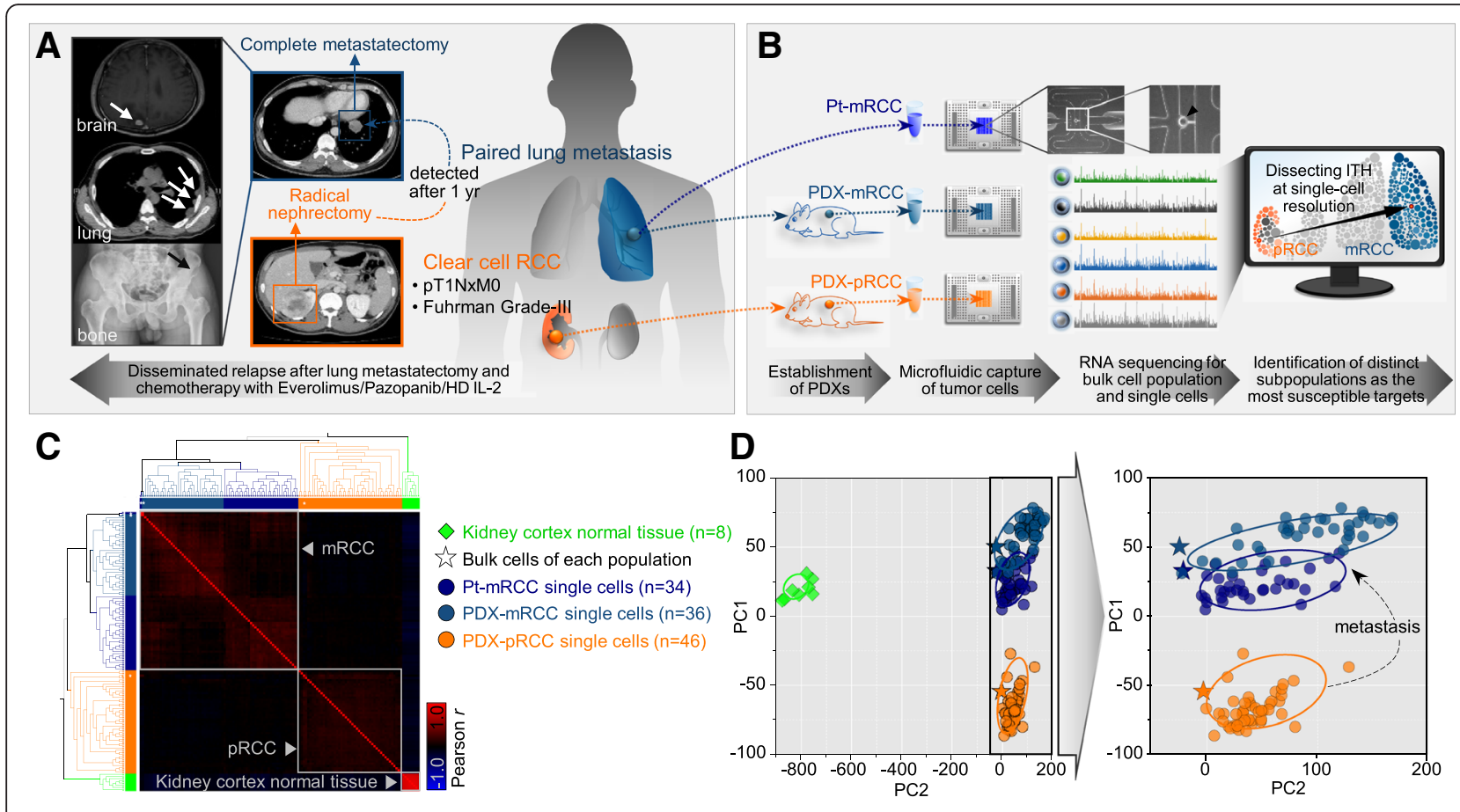

Fig. 1 Profiling transcriptome of paired pRCC and mRCC at single-cell resolution. a Brief description of clinical course in a patient with metastatic RCC. $\mathbf{b}$ Schematic of scRNA-seq experiments from establishment of the patient-derived xenograft model to discovery of targetable subpopulations. c Hierarchical clustering heatmap and dendrogram based on inter-correlation of centroid global expression profiles across kidney cortex normal, bulk cells of each population, and single cells using Euclidean distance metric and average linkage. $\mathbf{d}$ Principal component analysis (PCA) of single-cellresolved gene expression profiles based on the first two principal components. Ellipses represent $95 \%$ confidence around each group

mutations were shared at high cellular frequencies, whereas minor subclones were enriched in $\mathrm{mRCC}$ (Additional file 4: Figure S3A, B and Additional file 5: Table S2). Overall, the RCC of our patient showed a complex non-linear branching clonal evolution (Additional file 4: Figure S3C) that may become the basis of intratumoral diversity $[7,8,12]$. The genetic complexities might also result in molecular and functional differences between pRCC and mRCC despite their clonal origin, as previously reported [9-11].

\section{Single-cell RNA sequencing and quality assessment for expression profiling}

To model the functional heterogeneity and to identify specific subpopulations that are phenotypically relevant to drug responses, we used scRNA-seq to profile single cells from the parental mRCC and PDX mRCC and pRCC (Fig. 1b and see "Methods"). After filtering out poor-quality cells, a total of 116 tumor cells from the parental mRCC $(\mathrm{n}=34)$, PDX-mRCC $(\mathrm{n}=36)$, and PDX-pRCC $(n=46)$ were used in subsequent analyses (Additional file 6: Figure S4 and Additional file 7: Table S3). When compared to the normal kidney cortex, single cancer cells had much more variable gene expression as shown by the high coefficient of variation for averaged gene expression (Additional file 8: Figure S5A). Nonetheless, housekeeping genes, including glyceraldehyde 3-phosphate dehydrogenase $(G A P D H)$ and beta-actin $(A C T B)$, were stably expressed across single cells with low variation within (Additional file 8: Figure S5A) and across (Additional file 8: Figure S5B) cell populations, suggesting functional significance of the gene expression heterogeneity.

Compared to the normal kidney cortex, parental and PDX cancer cells demonstrated low stromal and high ccRCC gene expression signatures (Additional file 9: Figure S6). Interestingly, the global expression profiles of patient-mRCC and PDX-mRCC single-cell samples were more closely related than those of PDX-pRCC samples (Fig. 1c and Additional file 10: Figure S7). Principal component analysis (PCA) across all samples showed three distinctive main clusters (Fig. 1d); in addition to a cluster of normal kidney cortex, a cluster of parental mRCC cells and PDX samples were separated from a cluster of PDX-pRCC samples. As identified in unsupervised clustering of global expression across samples (Fig. 1c), we found the averaged expression of single cells correlated well with that of their bulk cell population samples (Additional file 10: Figure S7B). Furthermore, multiple regression analysis on the transcriptomes of different sized pools of single cells to those of bulk cell population samples showed better representation of the bulk cell population with increasing number of single cells 
(Additional file 10: Figure S7C). Similarly, in the PCA plot, bulk cell population samples almost matched single cells (Fig. 1d). As the quality assessments and unsupervised clustering analyses demonstrate the reliability of our data, we further explored the single-cell transcriptomes.

\section{Metastatic and aggressive molecular signatures in mRCC transcriptomes}

Consistent with the previous observation that PDX cells reflect their parental tumors with rare contamination of normal stromal cells [24], we verified discrete global expression profiles of single RCC cells compared with normal kidney expression profiles (Fig. 1c, d, Additional file 9: Figure S6, and Additional file 10: Figure S7). The stromal signature of parental bulk mRCC was higher than in other tumor samples (Additional file 9: Figure S6A), concordant with genomic analysis showing a smaller tumor portion in parental mRCC than PDX samples (Additional file 4: Figure S3B). By comparison, single cells from the parental mRCC all showed low stromal signatures (Additional file 9: Figure S6A) and stably higher ccRCC signatures (Additional file 9: Figure S6B), suggesting selective capture of tumor cells from the bulk cell populations. This might be explained by the experimental approach of using large size $(17-25 \mu \mathrm{m})$ microfluidic chips to capture single cells based on the histopathologic properties of the RCC tumor cells (Fuhrman Grade-III, average cell size $\sim 20 \mu \mathrm{m}$ ) [26] to reduce the likelihood of capturing stromal cells.

We then sought to identify distinct molecular signatures between $\mathrm{mRCC}$ and pRCC tumors. In recognition of the critical role of epithelial-mesenchymal transition
(EMT) in promoting metastasis $[27,28]$, we first examined the "EMT-induced" signature. In the virtual space of the PCA plot (Fig. 1d), the EMT-induced signature was enriched both in patient-mRCC and PDX-mRCC samples compared to PDX-pRCC samples (Additional file 11: Figure S8A) with statistical significance (Additional file 11: Figure S8B). Compared to the parental pRCC tumor, mRCC samples also showed elevated metastatic signatures (Fig. 2a and b). Because metastatic tumors tend to have worse prognostic properties and poor survival outcomes [29], we evaluated the "tumor aggressiveness" using prognostic markers that classify patients according to their survival rates [25]. Similar to the EMT-induced signature, we observed worse prognostic marker expression in mRCC cells, suggestive of tumor aggressiveness (Fig. 2c, d). From biological enrichment analysis with differentially expressed genes between pRCC cells and mRCC cells, Gene Ontology terms supporting tumor aggressiveness, such as "regulation of cell proliferation," "regulation of cell death," or "regulation of response to stress," were also enriched in mRCC cells (Additional file 12: Figure S9). Collectively, these results demonstrate distinct gene expression properties of $\mathrm{mRCC}$ cells, with enhanced metastatic and aggressiveness signatures compared to pRCC cells.

\section{Prediction of activation of drug target pathways and sensitivity to drug responses}

Distinct gene expression profiles in pRCC and mRCC tumor cells suggest divergent tumor cell behavior, including altered drug responses. Using predefined gene sets involved in drug target pathways, we estimated the

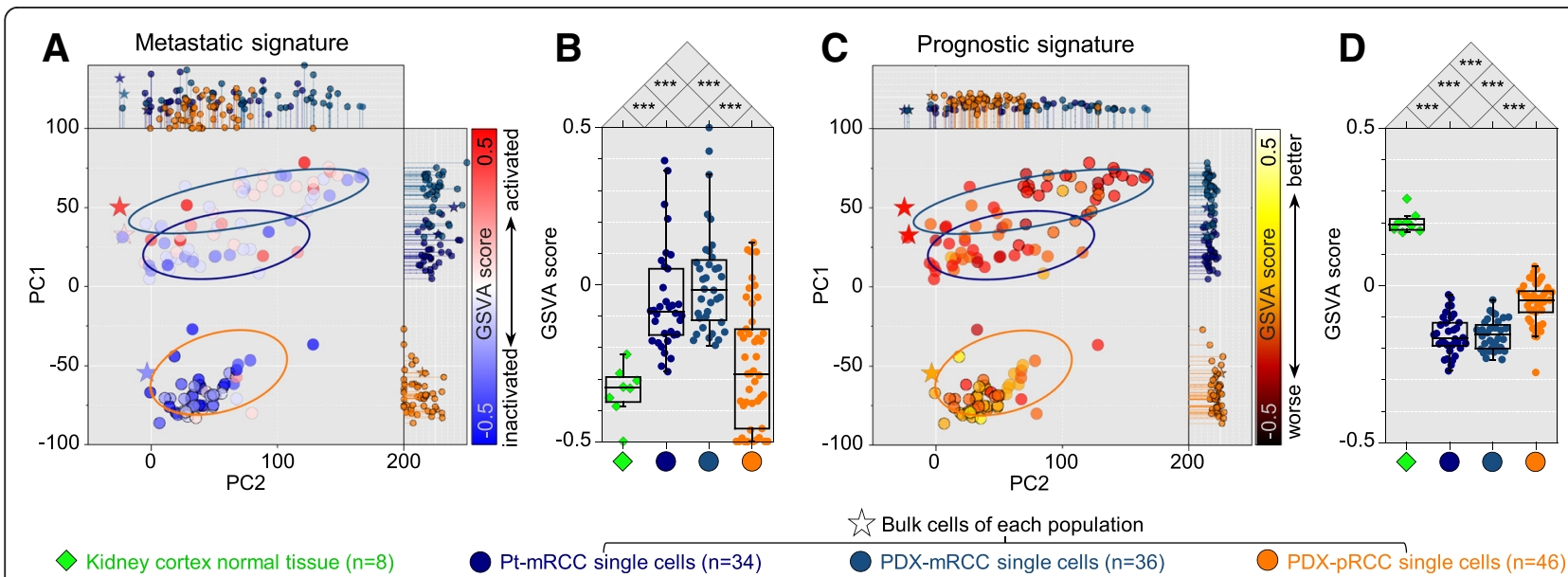

Fig. 2 Evaluation of expression signatures associated with metastatic RCC across single cells. a-d Gene set activation analysis for the metastatic signature ( $\mathbf{a}$ and $\mathbf{b}$ ) and clear cell RCC prognostic signature (c and $\mathbf{d}$ ). $\mathbf{a}, \mathbf{c}$ Positions of each dot and ellipse in Fig. 1d were fixed, and then each dot was colored (main panel) and indicated as drop lines (top and right panels) according to the estimated status for the given signatures. Gene Set Variation Analysis (GSVA) scores were normalized to normal kidney tissue expression profiles. b, d Boxplots show overall reciprocal differences in the expression signatures across normal kidney cortex, bulk cells of each population, and single cells. Boxes show 25th to 75th percentile with 10th and 90th percentile whiskers. ${ }^{*} P<0.05,{ }^{* *} P<0.01$, ${ }^{* *} P<0.001$, two-tailed Student's t-test 
relative activation status of the drug sensitivity signatures across PDX-mRCC and PDX-pRCC cells. Many drug target pathways were differentially regulated in the two PDX tumor cell groups (Fig. 3a and Additional file 13: Figure S10), suggesting differential drug sensitivity. We subsequently screened the PDX-mRCC and PDXpRCC cells with a panel of targeted agents (Fig. 3b and Additional file 14: Table S4). In repeated measures analysis for drug sensitivity, we observed nearly identical measurements in duplicate with a high statistical power
(Additional file 15: Figure S11). PDX-mRCC cells showed significantly higher expression of genes in the EGFR, Src, and BRAF/MEK pathways compared to PDX-pRCC cells, suggesting these pathways as mRCC-specific druggable targets. The PDX-mRCC cells showed ample responses to agents targeting EGFR (gefitinib, erlotinib, and afatinib), Src (dasatinib), and BRAF/MEK (selumetinib), substantiating the prediction. On the other hand, gene expression and activation scores in c-Met and PI3K/AKT pathways were significantly higher in PDX-pRCC cells. Concordantly,

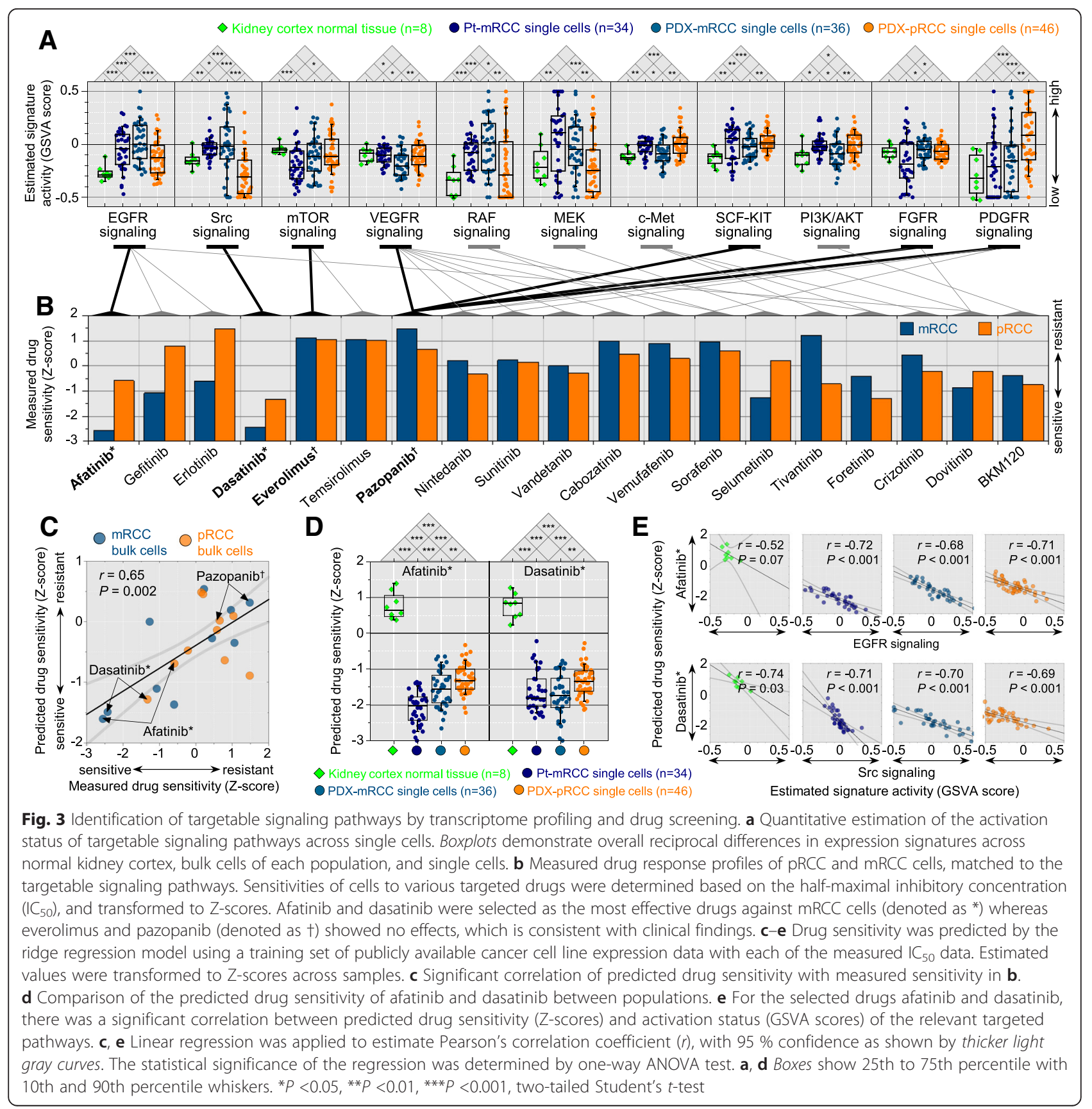


PDX-pRCC cells were more sensitive to agents targeting c-Met (tivantinib, foretinib, and crizotinib) and PI3K (BKM120) than PDX-mRCC cells.

To refine the predictive power of signaling pathway activation for drug sensitivity, we built a ridge regression model [30] using public gene expression profiles and drug sensitivity data as a training set [31]. For the PDXmRCC and pRCC bulk cell population samples, we found strong positive correlations between predicted and measured drug sensitivity (Fig. 3c). Drug sensitivity was also estimated in all single cancer cells and in normal kidney cortex as a control; these data suggested afatinib and dasatinib sensitivity of pRCC cells (Fig. 3d), similar to the predicted and measured drug sensitivity of bulk cell populations (Fig. 3b). The prediction of drug sensitivity by the ridge regression model was in agreement with the estimation of drug target pathway activation across single cells (Fig. 3e and Additional file 16: Figure S12). Interestingly, pazopanib and everolimus, which showed no clinical benefit in this patient (Fig. 1a), had minor effects on the viability of PDX-pRCC and PDX-mRCC cells (Fig. 3b and Additional file 17: Figure S13) and signaling pathway activation for their targets mTOR and VEGFR also remained low (Fig. 3a and Additional file 13: Figure S10). Although drug sensitivity of cancer cells was predicted to be higher than that of normal kidney cortex for all of the anticancer agents, the difference in pazopanib sensitivity was small (Additional file 16: Figure S12A). Overall, drug sensitivity predictions showed significant correlations with corresponding signaling pathway activation (Additional file 16: Figure S12B). We estimated the activation status of signaling pathways and drug sensitivity and demonstrated concordance between the predicted signatures and the measured data. These results suggest that molecular targeted therapies can be designed on the basis of prediction signatures obtained from RNA-seq.

\section{Heterogeneity in drug response signatures within a tumor}

Dysregulation of EGFR [32-34] and Src/FAK [35, 36] pathways plays a critical role in the tumorigenesis and metastatic progression of RCCs. The anticancer agents afatinib [37] and dasatinib [38, 39], targeting EGFR and Src signaling, respectively, have shown efficacy in the treatment of metastatic RCC. In drug sensitivity prediction and screens, PDX-mRCC cells showed the highest sensitivity to afatinib and dasatinib among the various drugs tested (Fig. 3b). At the single-cell level, PDX-mRCC showed extensive heterogeneity in the activation status of the EGFR and Src pathways (Fig. 3a and Additional file 13: Figure S10). This heterogeneous cellular pattern enabled us to classify cells according to the binary activation status of EGFR and Src signaling pathways (Fig. 4a), which was corroborated by the drug sensitivity prediction (Fig. 4b and Additional file 18: Figure S14). Notably, the classified cellular fractions were similar between the parental mRCC and PDX-mRCC (Fig. 4c), suggesting that heterogeneity in drug sensitivities for the PDX model reflects that of the parental tumor.

The heterogeneous drug sensitivity prediction for individual tumor cells suggests the presence of tumor subpopulations with distinct signaling pathway activation and drug sensitivity. Based on the prediction results, we identified four mRCC subpopulations with corresponding signaling pathway activation for EGFR and Src pathways (both active, only EGFR active, only Src active, both inactive; Fig. 4). Only a fraction of cells (23.5 \% in parental mRCC; $27.8 \%$ in PDX-mRCC) with activated status for both in EGFR and Src signaling pathways were predicted to be sensitive to both afatinib and dasatinib. A population with inactivated status in both signaling pathways (14.7\% in parental mRCC; 13.9 \% in PDX-mRCC) is unlikely to respond to afatinib or dasatinib. The largest proportion of cells $(61.8 \%$ in parental mRCC; $58.3 \%$ in PDX-mRCC) had signaling pathway activation in either of the two pathways, suggesting a mutually exclusive response to the corresponding drugs. This classification suggests that cotargeting of EGFR and Src with a combination of afatinib and dasatinib would be an efficient therapeutic strategy without redundancy and with additive antitumor effects (Fig. 4e).

\section{Evaluation of the single-cell analysis-driven therapeutic strategy}

Having recognized the existence of distinct subpopulations with differential drug sensitivities, we tested the efficacy of co-targeting EGFR and Src signaling pathways in PDX-mRCC cells. On the basis of transcriptome profiling and high-throughput drug screening (Fig. 3), we first measured the growth inhibitory effects of afatinib and dasatinib (Fig. 5a) in a two-dimensional (2D) culture system. Notably, compared to single drug treatment, the combination therapy more effectively suppressed the viability of mRCC cells (Fig. 5a). The efficiency of the combinatorial drug treatment was also examined in the spheroid formation model, which is a well-characterized three-dimensional (3D) culture and screening model with the advantages of simplicity, reproducibility, and similarity to physiological tissues compared with other methods involving extracellular matrix scaffolds and hydrogel systems [40, 41]. Consistent with the results obtained from the 2D system, we observed a superior effect of the combination therapy in the 3D system (Fig. 5b). Co-treatment with afatinib and dasatinib caused complete abrogation of EGFR and Src activity and more efficient inhibition of downstream AKT and 


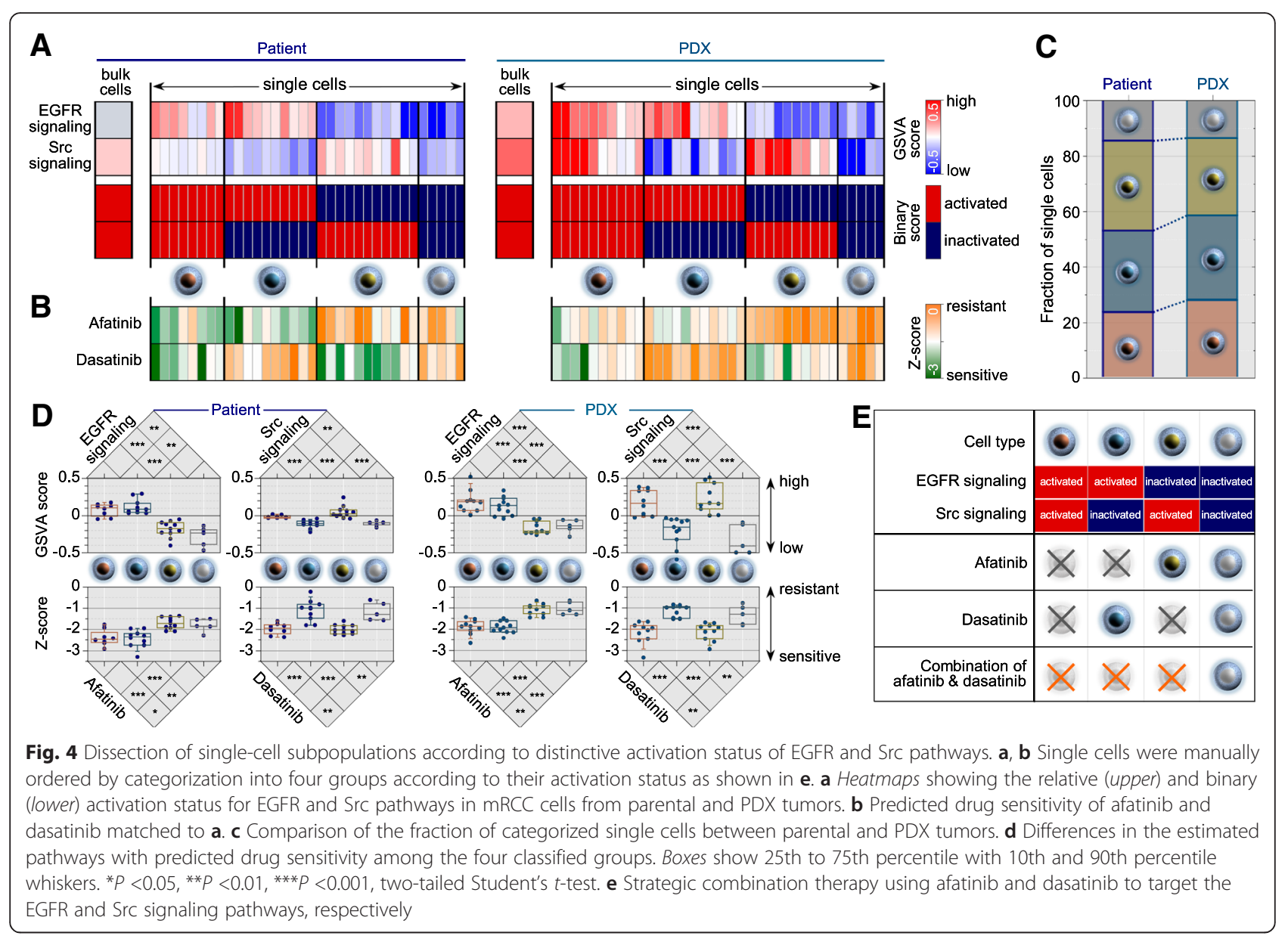

ERK phosphorylation in PDX-mRCC cells than that achieved by monotherapy (Fig. 5c).

Finally, we tested the in vivo performance of the combinatorial drug treatment using the subcutaneous xenograft model of mRCC. As single agents, afatinib $(20 \mathrm{mg} / \mathrm{kg})$ or dasatinib $(15 \mathrm{mg} / \mathrm{kg})$ had modest inhibitory effects on tumor growth (afatinib, $55 \%$; dasatinib, $40 \%$; Fig. 5d). Despite the partial treatment effect, the tumor cells continued to grow and none of the tumor xenografts showed complete response. In combination, afatinib and dasatinib showed a significantly enhanced antitumor effect, inhibiting tumor growth by $78 \%$ (Fig. 5d). Single-agent and combination treatment protocols were well tolerated by the mice, with no weight loss or other signs of acute or delayed toxicity (Fig. 5e). The drug effects were confirmed at the molecular level by complete inhibition of AKT (Fig. 5f) and ERK (Fig. 5g) phosphorylation. Together, co-targeting functionally distinct subpopulations in mRCC PDX cells that were identified by single-cell transcriptome analyses significantly improved treatment outcomes in both in vitro and in vivo investigational models.

\section{Discussion}

The evolution of multiple tumor subclones during tumor progression and metastasis generates intratumoral heterogeneity, which plays a role in intrinsic and acquired treatment resistance to molecular targeted therapies. In mRCC, durable complete responses are rarely achieved with conventional targeted molecular agents [1]. Furthermore, primary tumors and their corresponding metastases frequently show significant differences in therapeutic responses [13], suggesting that biopsies should be taken upon metastatic relapse for molecular profiling analysis to inform selection of salvage therapies. To date, most molecular profiling has been performed in the primary tumors of RCC patients and therefore failed to recapitulate the metastatic population. To overcome such clinical challenges, therapeutic strategies that efficiently target heterogeneous tumor subpopulations in $\mathrm{mRCC}$ must be developed.

In this report, we introduce scRNA-seq as a promising strategy that allows high-resolution analyses of the intratumoral landscape for tumor transcriptomes [42] and optimization of targeted treatment regimens 

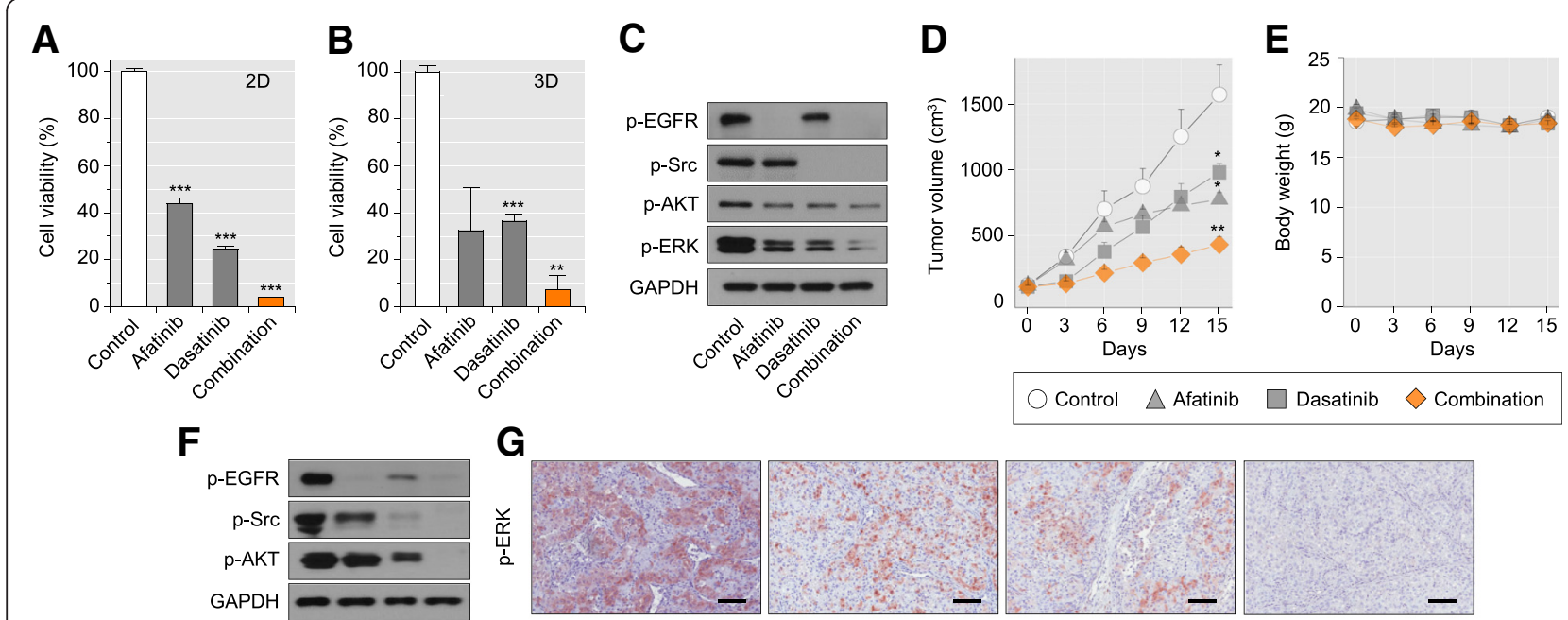

G
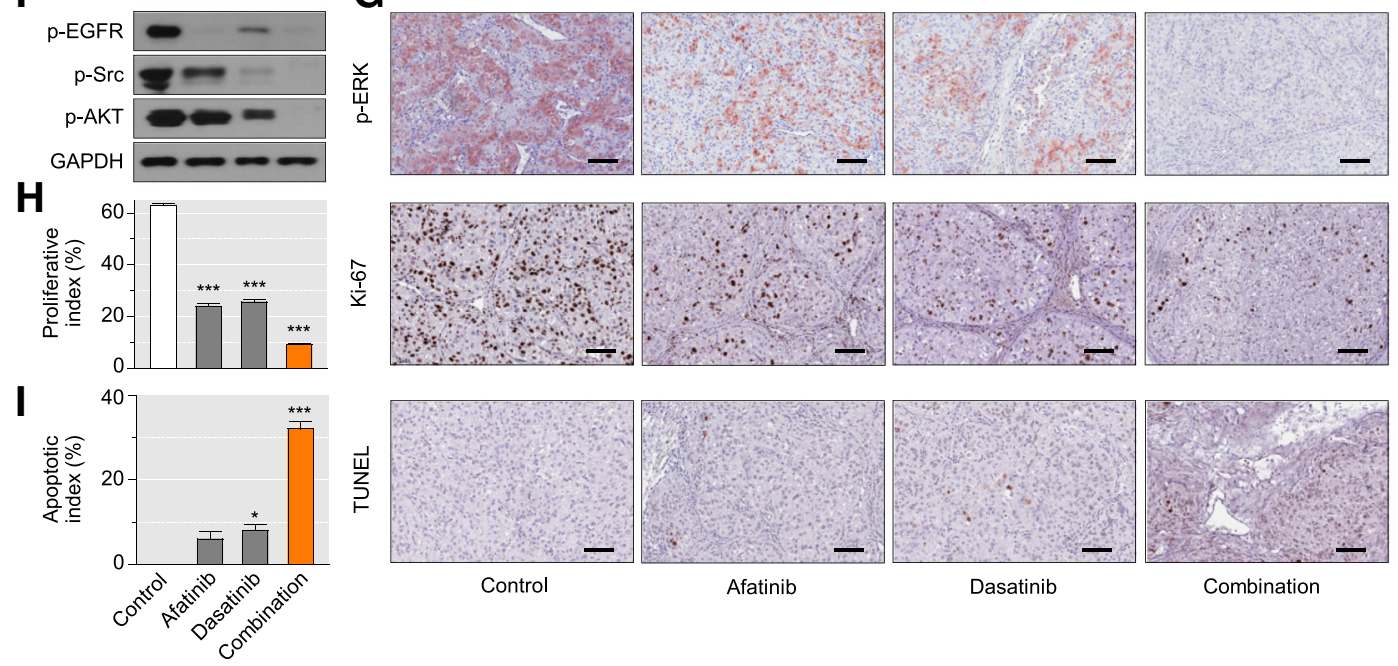

Fig. 5 Effective combinatorial strategy targeting heterogeneous subclones in mRCC. a, b Combined effects of afatinib and dasatinib on viability of mRCC cells were analyzed 6 days after treatment under 2D non-adherent culture conditions $\mathbf{a}$ or 3D ECM scaffold culture system $\mathbf{b}$. $\mathbf{c}$ Effects of afatinib and dasatinib combination therapy on EGFR and Src downstream pathways were validated by immunoblotting. Cells were incubated with $0.5 \mu \mathrm{M}$ afatinib and/or dasatinib for $1 \mathrm{~h}$. GAPDH = loading control. Cells that were mock-treated with $0.5 \mu \mathrm{M}$ DMSO served as negative controls. Error bar $=$ SEM ( $n=3$ for each group). ${ }^{* *} P<0.01,{ }^{* * *} P<0.001$. $\mathbf{d}-\mathbf{i}$ Superior antitumor efficacy of combinations of afatinib and dasatinib in mRCC subcutaneous xenografts. $\mathbf{d}$ Mice bearing $\mathrm{mRCC}$ tumors (=5 mice/group) were p.o. dosed with afatinib (each at $20 \mathrm{mg} / \mathrm{kg}$ ) or i.p. administered dasatinib (each at $15 \mathrm{mg} / \mathrm{kg}$ ), either alone or in combination, on a daily dosing regimen for up to 15 days. Growth curves based on tumor volumes are shown as the mean \pm SEM for each time point. ${ }^{*} P<0.05$, ${ }^{* *} P<0.01$. e Changes in body weight of mice treated with afatinib and/or dasatinib. Body weight was measured on the indicated days. Data show mean \pm SEM. Tumor tissues from each group were harvested on day 15 and subjected to immunoblot analysis with the indicated antibodies to detect p-EGFR, p-Src, and p-AKT (f; GAPDH = loading control), or immunostained with anti-p-ERK antibody $\mathbf{g}$. Proliferation and apoptotic rates in each group were determined by Ki-67 immunostaining $\mathbf{h}$ and TUNEL assay $\mathbf{i}$. Results are presented as mean values \pm SEM. ${ }^{*} P<0.05,{ }^{* * *} P<0.001$. Scale bars $=100 \mu \mathrm{m}$

against metastatic RCC. In our model case, we found that mRCC diverged from pRCC both at genomic and transcriptomic levels, manifesting distinctive genetic aberrations and drug target pathway activation. We combined comprehensive single-cell analyses with high-throughput drug screening in the PDX model and, based on these data, suggested a combinatorial treatment regimen targeting both EGFR and Src as the most efficient therapeutic option. In recognition of that drug responses are diverse in the pathologically identical tumors and even in the genetically homogeneous cancer cells $[16,17]$, profiling tumor transcriptome at the single-cell resolution can enable us to dissect inherent complex heterogeneous cell populations and to discern which cell would be different in drug responses affecting the ultimate outcomes that are covered under stochastic averaged signals in the bulk cell measurement. This strategy can be applied to other investigational models and eventually to patients with refractory cancer. Since higher engraftment rates are associated with more clinically aggressive tumors such as metastatic or treatment-refractory cases [43], surgically removed metastases or biopsy metastasis samples might extend the application of PDXs to advanced RCCs in terms of technical feasibility and unmet clinical needs.

Clonal genetic events in the metastases can be demonstrated for restricted subclones of the primary tumor, suggesting that only rare cells within the primary tumor have the ability to metastasize [44]. The parallel progression model proposes independent evolution of early disseminates in distant sites, eventually leading to significant 
divergence between the primary and metastatic tumors [45]. However, it remains largely unknown whether mRCCs also have initial ITH or whether the development of mRCCs is due to early dissemination or late diagnosis. Integrative analyses of PDXs derived from $\mathrm{pRCC}$ and distant metastasis to the lung from a single patient allowed us to infer which clonal and subclonal alterations contributed to tumor progression and spread to the metastatic lesion. The genetic differences between the pRCC and mRCC suggest highly complex non-linear branching clonal evolution as the basis for molecular and functional diversity, consistent with previous studies $[7,8]$. Moreover, key pathways that are targeted by clinically available drugs showed distinct expression patterns between pRCC and mRCC. Most driver aberrations and actionable driver mutations that have therapeutic implications were located on the branches, suggesting that distinct intratumoral subclones had acquired different functional characteristics [8]. The heterogeneity of metastatic cancer may underlie its poor responsiveness to therapy and explain why biomarkers of prognosis or therapy responsiveness measured exclusively from primary tumors give a restricted view of the biological properties of metastatic cancer [46].

Acquired resistance to therapy and disease progression can be due in part to intrinsic heterogeneity, including genetic, epigenetic, and biological properties of cancer cells that contribute to oncogenic activity. Thus, instead of single-agent therapy a rational approach that targets multiple subpopulations of tumor cells with a combination of non-cross-resistant drugs characterized by different mechanisms of action and non-overlapping profiles of toxicity will be necessary for long-lasting inhibition of highly heterogeneous mRCC $[7,8]$. Knowledge of molecular alterations and features of tumors and the identification of mechanisms of tumor resistance provide the opportunity to test novel rationally designed drug combinations. Recent technological advances in singlecell sequencing have facilitated the paradigm shift in our understanding of the cancer ecosystem from the averaged signal of a complex tumor mass to the sum of distinctive signals in individual cells [42]. Unique cellular behaviors reflecting oncogenic signatures have been extensively scrutinized by profiling transcriptomes from individual cells in various types of cancers [24, 47-49]. In the light of the precedent approaches for deconvolving heterogeneous cell populations and identifying specific cell types that are generally masked in bulk cell profiling, we could understand better the biologically relevant composition of cancer cells and their functional modulation within the tumor. Importantly, in this study such dissected intratumoral landscape enabled us to elicit the most effective drug combination of putting all the potentially targetable cancer cells together to be killed.

\section{Conclusion}

Using scRNA-seq, we could examine the heterogeneous drug target pathway activations at the single-cell level in a refractory $\mathrm{mRCC}$ patient. Distinct features of intratumoral expression variability across $\mathrm{mRCC}$ single cells that were masked in the bulk measurement prompted us to test the co-targeting strategy for the most vulnerable two signaling pathways with increased likelihood of complete response. Indeed, we observed significantly better treatment effects of the targeted combination therapy on mRCC-derived xenograft platforms in vitro and in vivo than monotherapies. Our findings described here will have a profound impact on translational research to overcome ITH-derived resistance and avoid ineffectual or unnecessary treatments. Although we could not analyze clinical response to our combination strategy because of rapid deterioration of the patient, we stress the utility and validity of single-cell transcriptome profiling in patients with refractory cancer for the design of personalized therapeutic strategies. In summary, the realization of the advantages in dissecting heterogeneous drug target pathway activations by scRNA-seq analysis will have significant clinical utility for the design of tailored combination therapy against highly heterogeneous tumors.

\section{Methods \\ Ethics}

This study was carried out in accordance with the principles of the Declaration of Helsinki, and was approved by The Samsung Medical Center (Seoul, Korea) Institutional Review Board (IRB) (no. 2010-04-004). Participants in this study gave written informed consent for the research and publication of the results. Animal experiments were conducted in accordance with the Institute for Laboratory Animal Research Guide for the Care and Use of Laboratory Animals and the following protocols were approved by the IRB at the Samsung Medical Center (Seoul, Korea) (No. 20131217002). Animal care and handling was conducted in accordance with the National Institute of Health Guide for the Care and Use of Laboratory Animals (NIH publication no.80-23, revised 1978).

\section{Sample selection and clinical characteristics of the patient} PDXs were established using surgically resected matched pRCC (pT1Nx; Fuhrman Grade 3) and paired lung metastasis from a 43-year-old man with ccRCC who experienced solitary lung metastasis 1 year after radical nephrectomy (IRB number 2010-04-004). He showed signs of rapid tumor dissemination to the bone, lung, pleura, and brain despite multiple salvage regimens, including pazopanib, everolimus, and high-dose interleukin (IL)-2, and finally died 16 months after complete metastatectomy as a result of rapid tumor progression. 
Serial 5- $\mu \mathrm{m}$ sections from each formalin-fixed paraffinembedded block were processed for hematoxylin and eosin (H\&E) staining and examined by a specialized pathologist.

\section{Establishment of orthotopic PDXs}

Athymic nude mice were obtained from Orient Bio (Seoul, Korea). Fresh tumor tissue was obtained from pRCC tissue of the patient by surgical excision under sterile conditions and matched fresh tumor tissue was taken from the mRCC. Each biopsied parental tumor mass was chopped into fragments, and was frozen or placed in formalin and embedded in paraffin for later analyses. A blood pellet was used for extraction of germline DNA. Fresh tumor tissue was stored on ice in Hank's Balanced Salt Solution (Gibco, Grand Island, NY, USA) supplemented with penicillin/streptomycin (Gibco) for transport. For transplantation, 6- to 8-week-old NOD scid gamma mice were anesthetized with $100 \mathrm{mg} / \mathrm{kg}$ ketamine and $10 \mathrm{mg} / \mathrm{kg}$ xylazine. Primary tumor and paired lung metastasis samples minced into approximately $1-\mathrm{mm}^{3}$ fragments in high-concentration Matrigel TM Basement Membrane Matrix (BD Biosciences, Franklin Lakes, NJ, USA) were directly implanted into the subrenal capsule ( $n=4-5$ for each tumor sample). For subrenal capsule implantation, xenograft tumor engraftment was defined as a palpable mass of $>1 \mathrm{~mm}$ in diameter with pathologic confirmation. When the resulting tumors grew, each tumor (F1 generation) was resected as the primary tumor, divided, and passaged into five mice (F2 generation). The PDX tumors were harvested and divided into three samples for generation of second in vivo passage xenograft tumors, DNA/RNA extraction, and histopathologic examination. The origin of each xenograft was validated by short tandem repeat DNA fingerprinting. The process was repeated to produce subsequent generations via subcutaneous implantation in BALB/c nude mice to expand xenograft numbers.

\section{Whole-exome sequencing}

We extracted genomic DNA from patient-derived tumor samples using the QIAamp DNA Minikit (Qiagen, Hilden, Germany) and from matched blood using the QIAamp DNA Blood kit (Qiagen). Purified DNA was sheared to an average size of $150 \mathrm{bp}$ in a Covaris Adaptive Focused Acoustics $^{\text {Th }}$ (AFA) sonication device (S2, Covaris, Inc., Woburn, MA, USA) and indexed with unique barcode tags using PCR. Prepared libraries were assessed for quality and quantity using a Qubit 2.0 Fluorometer (Life Technologies, Carlsbad, CA, USA), 2100 Bioanalyzer (Agilent Inc., Palo Alto, CA, USA), and Mx3005P qPCR (Agilent Technologies, Inc). Exomes were targeted using the SureSelect XT Human All Exon V5 kit (Agilent). Samples were multiplexed and flow-cell clusters were created using the TruSeq Rapid Cluster kit and TruSeq Rapid SBS kit (Illumina, San Diego, CA, USA). Captured exomes were sequenced using the Illumina HiSeq 2500 platform, and paired-end 100-bp sequence data were generated. Sequencing reads that only mapped to the mouse genome reference (mm10) were filtered out.

\section{Array comparative genomic hybridization}

Purified DNA from patient-derived tumor samples was labeled with Cy5-dUTP following the Agilent Oligonucleotide Array-Based CGH for Genomic DNA Analysis protocol (Ver-7.3, Agilent). Cy5-labeled DNA was quantified together with reference DNA samples labeled with Cy3-dUTP to determine the DNA concentration using an ND-1000 Spectrophotometer (NanoDrop, Wilmington, DE, USA). Labeled test and reference samples were then hybridized to SurePrint G3 Human CGH $4 \times 180 \mathrm{~K} \mathrm{Mi-}$ croarrays (Agilent) according to the manufacturer's standard protocol. The dual-colored fluorescence signals were scanned using the Agilent Microarray Scanner and translated to $\log 10$ ratios using Feature Extraction software (Ver-11.0.1.1, Agilent).

\section{Identification of single-nucleotide variants and copy number variants}

Using the WES data, generated reads were aligned to the human genome reference hg19 using the BurrowsWheeler Aligner [50] after removing duplicate reads, followed by implementation of the data-processing modules using the Genome Analysis Toolkit [51]. Somatic SNVs were identified by Bayesian statistical analysis of bases and their qualities in the given tumor and paired normal BAM files at the genomic locus using the MuTect algorithm [52]. Called variants were reviewed manually using the Integrative Genomic Viewer [53], and considered only within chromosomes 1-22 and $\mathrm{X}$. Mutations were annotated using the SnpEff [54] package.

Based on the CGH data, extracted signals were normalized to $\log 2$ ratios using the limma package [55]. To detect significant breakpoints across thousands of probederived signals, we applied the circular binary segmentation (CBS) algorithm using the DNAcopy package [56]. After smoothing the data to detect outliers within autosomal chromosomes, aberrant segments were determined applying the significance level of 1.0E-04 to accept change-points based on a maximum t-statistic. We classified the segmented results into copy losses when the $\log 2$ ratios were lower than -0.25 and copy gains when these were greater than 0.25 . Considering sample-specific tumor purity and ploidy, somatic copy number alterations (SCNA) were adjusted by implementing the ABSOLUTE algorithm [57]. To compare the 
SCNA patterns across samples, segment values were averaged with 1-kb binning along the chromosomes.

\section{Clustering of genomic clones}

To determine the subclonal structure between primary RCC and paired lung metastasis, we adopted the PyClone algorithm [58] that computes the cellular prevalence of mutations and clusters these mutations based on a hierarchical Bayes statistical model. Mutational information, including somatic SNVs called from the deep exomes and absolute copy-number changes corresponding to SNV regions, was prepared for use with PyClone. The cellular prevalence for each mutation was estimated using a beta-binomial model by setting the number of Markov chain Monte Carlo (MCMC) iterations to 100,000 , with a burn-in of 50,000. The number of clusters was inferred based on the average linkage hierarchical clustering in the post-burn-in trace by optimizing the maximization of posterior expected adjusted Rand index criterion.

\section{Single-cell RNA sequencing and processing}

scRNA-seq and data processing were carried out as previously described [24]. Briefly, cells isolated from human mRCC, PDX-mRCC, and paired PDX-pRCC tumors were subjected to single-cell capture and cDNA amplification using the $\mathrm{C}^{\mathrm{m}}$ Single-Cell Auto Prep System (Fluidigm, South San Francisco, CA, USA) with the SMARTer kit (Clontech, Mountain View, CA, USA). RNA-seq reads for single cells and bulk samples were generated using the HiSeq 2500 in the 100-bp paired-end mode and reads that only mapped to the mouse genome reference (mm10) were subsequently removed. Filtered reads were aligned to the human genome reference hg19 with the sample specific-splice junction using the 2-pass mode of STAR (Ver-2.4.0d) [59]. Transcripts per million (TPM) was quantified using RSEM (Ver-1.2.18) [60]. To filter out poor quality cells, we applied the critea of $>1 \mathrm{M}$ reads per cell, $>60 \%$ uniquely mapped rate, $>35 \%$ exonic region coverage rate, and $>5000$ detected genes. We considered TPM values greater than 1 to be reliable and only focused on genes that were detected in more than $10 \%$ of a group between pRCC and paired lung metastasis. To identify differential and common expression signatures between pRCC and paired lung metastasis single cells compared to normal signals, we used expression profiles of normal kidney cortex from the GTEx portal (http://www.gtexportal.org/home/; transcript read counts V3) by converting to TPM values. Finally, we normalized sampleto-sample variation by applying a mean centroid.

\section{Estimation of activity for expression signatures and drug} sensitivities

To understand the relative activation status for a pathway or signature across samples, we implemented the GSVA algorithm [61] in RNA-seq data. GSVA scores were estimated for given gene sets from the following sources: stromal signature, extracted from the ESTIMATE package [62]; ccRCC signature, taken from Jones et al. [63]; EMT-induced signature, taken from Taube et al. [64]; metastatic signature, taken from Jones et al. [63]; prognostic signature, taken from The Cancer Genome Atlas Research Network for ccRCC [25]; EGFR signaling, (Reactome, signaling by constitutively active EGFR); Src signaling, extracted from Gatza M.L. et al. [65]; mTOR signaling (Reactome, mTOR signaling); VEGFR signaling (PID, signaling events mediated by VEGFR1 and VEGFR2); RAF signaling, (Reactome, RAF activation); MEK signaling, (Reactome, MEK activation); c-Met signaling, (PID, signaling events mediated by c-Met); SCFKIT signaling, (Reactome, signaling by SCF-KIT); PI3K/ AKT signaling (Reactome, PI3K/AKT signaling in cancer); FGFR signaling (Reactome, signaling by FGFR); PDGFR signaling (PID, PDGF receptor signaling network). To evaluate whether an estimated gene set signature is significantly activated, we transformed the observed GSVA scores to binary scores. Gene sets with the same size as each original panel of genes were randomly generated with permutation $(\times 1000)$ and computed for their GSVA scores. Assignment of the original GSVA scores as "activated" was determined with the cutoff values of averaged scores in the randomly selected gene sets.

In addition to estimation of activity status for signaling pathways, relevant targeting drug sensitivities were also predicted from expression profiles. Following a previous approach [30], cancer cell line expression data with measured drug response data from the Cancer Genome Project (CGP) [31] were used as a training set. After adjusting two independent sets of our expression data with the training set of solid tumor cell lines using ComBat [66], a ridge regression model was fitted for the training set with the given drugs that were simultaneously identified in our study. The lowest varying $20 \%$ of genes were filtered out to focus on biological variability over technical variability. To evaluate the prediction sensitivity, leave-one-out-cross-validation (LOOCV) was applied with the total dataset. From this computation for a total of 10 drugs (BIBW2992 [Afatinib], Dasatinib, Gefitinib, Erlotinib, Temsirolimus, Pazopanib, Sunitinib, Sorafenib, AZD6244 [Selumetinib], and PF-02341066 [Crizotinib]), our data were tested to predict drug sensitivity and the distribution of estimates was transformed to Z-scores by dividing the averaged drug sensitivity estimates by the standard deviation for the difference. 
To determine the prediction accuracy of drug sensitivity estimates with measured drug sensitivity from highthroughput drug screening, nanomolar scaled $\mathrm{IC}_{50}$ values were also transformed to Z-scores.

\section{Functional network analysis}

To identify discrete biological networks respectively enriched in pRCC and mRCC cells, differentially expressed genes (DEGs) were first defined with a criteria of the Benjamini-Hochberg corrected FDR $<0.01$ and fold changes of at least twofold. DEGs were separately applied to Gene Ontology (GO) category analysis using the ClueGO [67] plug-in within the Cytoscape framework [68]. The statistical significance for the over-represented pathways in the GO Biological Process category was estimated using Benjamini-Hochberg correction for multiple testing-controlled $P$ values. Significantly enriched terms were functionally grouped based on kappa scores $>0.3$.

\section{Primary in vitro short-term culture}

Xenograft tumor specimens were dissociated into single cells according to previously published protocols [69]. Dissociated PDX cells were cultured in neurobasal media-A supplemented with N2 ( $\times 1 / 2$, Life Technologies, Carlsbad, CA, USA), B27 $(\times 1 / 2$, Gibco), basic fibroblast growth factor (20 ng/mL; R\&D Systems, Minneapolis, MN, USA), epidermal growth factor (EGF, $20 \mathrm{ng} / \mathrm{mL}$; R\&D Systems), neuregulin 1 (10 ng/mL; R\&D Systems), and insulin-like growth factor 1 (100 ng/mL; R\&D Systems) and containing $10 \%$ conditioned medium (CM) from human mesenchymal stem cells (MSCs). To generate the CM, MSCs were grown to $70 \%$ confluency in plates with $10 \%$ FBS L-DMEM and allowed to adhere overnight at $37^{\circ} \mathrm{C}$ and $5 \% \mathrm{CO}_{2}$. The following day, the medium was replaced with serum-free culture medium and the cells were cultured for 2 days. The used medium was collected as MSC-CM, centrifuged to remove cell debris, and passed through a $0.45-\mu \mathrm{m}$ filter. $\mathrm{CM}$ aliquots were frozen at $-80{ }^{\circ} \mathrm{C}$ until use.

\section{In vitro drug sensitivity assay}

Primary RCC PDX cells cultured under serum-free sphere culture conditions were seeded in 384-well plates at 500 cells per well. Two hours after plating, the cells were treated with a drug library in threefold and 10point serial dilution series ( $\mathrm{n}=3$ for each condition). After incubation at $37{ }^{\circ} \mathrm{C}$ in a $5 \% \mathrm{CO}_{2}$ humidified incubator for 6 days, cell viability was analyzed using an adenosine triphosphate monitoring system based on firefly luciferase (ATPliteTM 1step, PerkinElmer, Waltham, MA, USA). The drug library was composed of 20 targeted agents that were included in the clinical guidelines or in current clinical trials (gefitinib, erlotinib, lapatinib, afatinib, tivantinib, foretinib, crizotinib, selumetinib, temsirolimus, everolimus, cabozantinib, vandetanib, sunitinib, sorafenib, dovitinib, vemurafenib, BKM 120, pazopanib, nintedanib, and DAPT; all purchased from Selleckchem, Houston, TX, USA). The drugs were stored and diluted according to the manufacturer's instructions. Test concentrations for each drug were empirically derived to produce a clinically relevant spectrum of drug activity. Half-maximal (50\%) inhibitory concentration values $\left(\mathrm{IC}_{50}\right)$ were calculated as an average of triplicate experiments using the S+ Chip Analyzer (Samsung ElectroMechanics Company, Ltd., Gyeonggi, Korea) [70].

For signal transduction assays under treatment with the targeted agents, primary cultured PDX cells were maintained overnight in serum-free sphere culture conditions without growth factors, incubated for $1 \mathrm{~h}$ with each inhibitor, and pulsed with original culture medium supplemented for $15 \mathrm{~min}$. For immunoblotting, cells were lysed in RIPA lysis buffer supplemented with $1 \times$ phosphatase inhibitors (PhosStop; Roche Diagnostics, Basel, Switzerland) and a $1 \times$ protease inhibitor cocktail (Complete Mini; Roche Diagnostics). After centrifugation at $10,000 \times g$ for $5 \mathrm{~min}$, the supernatant was harvested and protein concentration was determined using a bicinchoninic acid protein assay kit (Thermo Scientific, Waltham, MA, USA). Equal amounts of protein were subjected to SDS-PAGE and transferred to polyvinylidene difluoride membranes (Whatman plc, Little Chalfont, UK). Membranes were blocked in $5 \%$ skim milk or bovine serum albumin for $1 \mathrm{~h}$ at room temperature, incubated with the indicated primary antibodies overnight, and then with the appropriate secondary antibodies. Antibodies against p-EGFR (Tyr1068), p-Src (Tyr527), p-ERK, p-AKT (Ser473) (all purchased from Cell Signaling Technology, Danvers, MA, USA), and GAPDH (Santa Cruz Biotechnology, Santa Cruz, CA, USA) were used.

\section{Preparation of microfluidic drug screening device}

The microfluidic drug screening device was made of polydimethylsiloxane (PDMS, Sylgard 184; Dow Corning, Corning, NY, USA) using a conventional softlithography process with 200 micron high SU-8 patterned silicon wafer (MicroChem, Westborough, MA, USA). The fabricated device was sterilized and bonded onto a cover glass to enclose the microchannels by oxygen plasma treatment (Femto Science, Somerset, NJ, USA). The device was then heated in an oven at $80{ }^{\circ} \mathrm{C}$ for $24 \mathrm{~h}$ to allow the surface to recover its hydrophobicity. The restored hydrophobicity of the microfluidic channel surface helps the injected extracellular matrix (ECM) form a stable interface with the side channels. In repeated measures analysis for drug sensitivity, the identical experimental condition was applied to the second measurement in PDX-mRCC cells. 
Three-dimensional cell culture and drug treatment Collagen type 1 (3 mg/mL, rat tail; Corning) was used as an ECM scaffold to embed the cells. The collagen solution was prepared at $\mathrm{pH} 7$ and a concentration of $2 \mathrm{mg} / \mathrm{mL}$. This solution was diluted in a mixture of $10 \times$ phosphate-buffered saline (PBS; Gibco) and sterilized deionized water. The $\mathrm{pH}$ was adjusted using $0.5 \mathrm{~N} \mathrm{NaOH}$. Dissociated cells were suspended in the collagen solution at a density of $0.5 \times 10^{6}$ cells $/ \mathrm{mL}$. The suspension was injected into a center channel of the device and allowed to gel by incubation at $37{ }^{\circ} \mathrm{C}$ and $5 \% \mathrm{CO}_{2}$ for $30 \mathrm{~min}$. Details of the device preparation and gel filling procedure have been described previously [41]. To avoid cell attachment to the microfluidic channel surface, the device was inverted every $5 \mathrm{~min}$. After gel formation, the side channels were filled with medium containing each drug candidate. The medium in the channel was refreshed every $24 \mathrm{~h}$.

\section{Live/dead assay}

Cell viability was quantified at 4 and 7 days of culture using the Live/Dead Viability Assay Kit (Molecular Probes, Invitrogen, Carlsbad, CA, USA) containing calcein $\mathrm{AM}$ and ethidium homodimer to identify live (green) and dead (red) cells, respectively. Cells in the microfluidic device were incubated at $37{ }^{\circ} \mathrm{C}$ with $5 \% \mathrm{CO}_{2}$ for $30 \mathrm{~min}$, and then the staining solution was replaced with PBS. The cells were counted using ImageJ software (Image Processing and Analysis in Java, NIH, Bethesda, MD, USA). Cell viability was calculated as the number of live cells divided by the total cell number. Normalized cell viability was determined by dividing cell viability by the viability of cells cultured in pure medium condition.

\section{In vivo drug efficacy}

$\mathrm{BALB} / \mathrm{c}$ nude mice (female, 6-8 weeks old; Orient Bio) were used for drug efficacy studies. Animal experiments were conducted in accordance with the Institute for Laboratory Animal Research Guide for the Care and Use of Laboratory Animals and the following protocols were approved by the IRB at Samsung Medical Center (Seoul, Korea). Briefly, $2 \times 10^{5}$ dissociated PDX-mRCC cells mixed 1:1 with Matrigel (BD Biosciences) were inoculated subcutaneously into the right flank of each mouse. Tumor diameters were measured with calipers twice a week and tumor volume in $\mathrm{mm}^{3}$ was calculated using the following formula: tumor volume $=\left(1 \times w^{2}\right) / 2$, where 1 is the longest diameter of the tumor and $w$ is the shortest diameter of the tumor. Mice bearing established tumors $\left(100-150 \mathrm{~mm}^{3}\right)$ were randomly allocated to four groups (5 in each group): vehicle, afatinib at $20 \mathrm{mg} / \mathrm{kg}$ (p.o.), dasatinib $15 \mathrm{mg} / \mathrm{kg}$ (i.p.), or afatinib/ dasatinib combination on a daily dosing regimen for up to 15 days. Mice in the four groups exhibited similar average tumor volumes and body weight. Throughout the study, the mice were weighed and tumor burden was monitored every 3 days. Mean tumor volumes were calculated, and growth curves were established as a function of time. The error bars indicate the value of the standard error of the mean (SEM). Tumors from each group were collected at the end of the experiment for further analysis.

\section{Immunohistochemical and TUNEL staining}

Tumors were embedded in paraffin, sectioned at $5 \mu \mathrm{m}$, and stained with H\&E. Paraffin-embedded tissue sections were deparaffinized and rehydrated. For immunochemical staining, heat-induced epitope retrieval was performed using a target retrieval solution (Dako, Glostrup, Denmark) for $5 \mathrm{~min}$ in a microwave. Slides were treated with $3 \%$ hydrogen peroxide for $10 \mathrm{~min}$ to inactivate endogenous peroxidase and then blocked for $20 \mathrm{~min}$ at room temperature (RT) in blocking solution (5\% normal horse serum, $1 \%$ normal goat serum, $0.1 \%$ Triton-X 100 in $1 \times$ PBS). After blocking, the slides were incubated with primary antibodies, including mouse monoclonal antibody against p-ERK (Cell Signaling Technology) and human Ki-67 (BD Pharmingen). After washing, the slides were incubated with secondary antibodies for $1 \mathrm{~h}$ at RT, and counterstained with hematoxylin (Sigma-Aldrich, St. Louis, MO, USA). Apoptotic cells were identified by histologic analysis of DNA fragmentation in paraffin sections of the xenograft tumors. We performed terminal deoxynucleotide transferase-mediated dUTP nick end labeling (TUNEL) staining on the tumor sections using the DeadEnd ${ }^{\text {th }}$ Colorimetric TUNEL System (Promega, Madison, WI, USA). The proliferative or apoptotic index was calculated as a ratio of Ki-67-positive or TUNEL-positive cell number to total cell number in high-power $(\times 400)$ fields.

\section{Statistical analysis}

All values are expressed as the mean \pm SEM. Comparisons between two groups were analyzed by Student's $t$-test. One-way analysis of variance was applied for comparisons between more than two groups and to determine the statistical significance for the fitting model in the linear regression of two components. All $P$ values were two-sided, and $P<0.05$ was considered statistically significant. For discovery of differentially expressed genes (DEGs) between pRCC and mRCC cells, we applied the Benjamini-Hochberg correction for multiple-testing. Statistically significant DEGs were regarded using the cutoff of FDR $<0.01$ and foldchange $\geq 2$. Multiple regression analysis was applied to evaluate the transcriptomic heterogeneity across single cells, and to estimate the explanatory power of randomly selected single cells (with the given number by 
permutation $\times 1000$ ) attributed to the expression profile of the bulk measurement by calculating the adjusted R-square. All data analyses were performed using SPSS statistical software, version 19.0 (SPSS, Inc., Chicago, IL, USA).

\section{Data access}

RNA sequence and aCGH data have been deposited in the National Center for Biotechnology Information (NCBI) Gene Expression Omnibus (GEO) under accession number GSE73122. Whole-exome sequence data can be accessed at the NCBI Sequence Read Archive (SRA) with accession number SRP063388.

\section{Additional files}

Additional file 1: Figure S1. Histologic and genetic similarity between parental tumor and PDX model. A Morphologic similarity between parental tumors and matched xenografts by hematoxylin and eosin (H\&E) staining and bright-field observation for tumor tissues and PDX cells, respectively. B Tumor purity and ploidy of the PDX and parental tumors were computationally estimated using the ABSOLUTE algorithm. C Somatic single-nucleotide variants (SSNVs) of the PDX and parental tumors are compared in a Venn diagram. The number of SSNVs shared by all four tumors is colored yellow. D Changes in cellular prevalence of SSNVs are presented. SSNVS that were shared by all four tumors $(n=59)$ are highlighted by yellow outlined circles and thick lines. Non-silent SSNVS common in ccRCC (Additional file 2: Figure S2C) are denoted. E Somatic copy number alterations (SCNAs) along the autosomal chromosomes of the PDX and parental tumors were adjusted with estimated tumor purity and ploidy and presented as heatmaps. The order of tumors was determined by average linkage clustering in Euclidean distance similarity metrics. Common SCNAs of CCRCCs are identified in Additional file 2: Figure S2B. F Pearson correlation coefficients ( $r$ ) of copy number profiles between samples are presented. (PDF $2.57 \mathrm{mb}$ )

Additional file 2: Figure S2. Comparative genetic profiles of paired pRCC and mRCC. A Left, variant allele frequencies (VAF) of somatic single-nucleotide variants (SSNVs) in pRCC and $\mathrm{mRCC}$ are presented as a dot plot. Gray, orange, and blue represent shared, pRCC-exclusive, and mRCC-exclusive SSNVs, respectively. Center, numbers of exclusive and shared SSNVs are shown in a Venn diagram. Right, boxplots demonstrating VAF distributions of exclusive and shared SSNVs of pRCC and MRCC. Boxes show 25th to 75th percentile with 10th and 90th percentile whiskers. Two-tailed Student's $t$-test was applied to determine statistical significance of differences in VAF distribution. For a full list, see Additional file 3: Table S1. B Array comparative genomic hybridization (aCGH) copy number profiles of pRCC and mRCC. Break points of CGH probes were detected using the circular binary segmentation (CBS) algorithm. Regions of differential copy number between pRCC (orange) and mRCC (b/ue) are shadowed with arrows along the chromosomes. CBS-derived copy gains (red) and losses (b/ue) were defined using a log2 ratio cutoff of positive 0.25 and negative 0.25 , respectively. C Somatic copy number alterations (SCNAs) and SSNVs of pRCC and MRCC were compared the common SCNA and SSNV sites of clear cell RCC (cCRCC). Filtering criteria for SSNVS and SCNAs are indicated. Only non-silent SSNVs are annotated. (PDF $484 \mathrm{~kb}$ )

Additional file 3: Table S1. Somatic mutations identified in tumor samples from whole-exome sequencing. Single-nucleotide variants (SNVs) were annotated by implementing SnpEff. Whole-exome sequencing data of paired patient blood was used to identify somatic variants in parental tumors and matched xenografts of pRCCs and mRCCs, which are shown in separate sheets. (XLSX $218 \mathrm{~kb}$ )

Additional file 4: Figure S3. Clonal evolution of RCC during metastatic spread to lung. Gaussian kernel density estimates were applied in a post- burn-in of Markov Chain Monte Carlo (MCMC) traces in the PyClone algorithm. A Total SSNVs are annotated using the official gene symbol with chromosomal position and non-silent mutations are highlighted in bold. Arrows indicate underlying driver mutations in Additional file 2: Figure S2C. B Mean cellular prevalence of each SSNV (for full list, see Additional file 5: Table S2). Dominant subclones harboring SSNVs with higher cellular prevalence are ordered starting from the left. C Inferred phylogenetic evolutionary pattern between PRCC and mRCC. Branch and trunk lengths are proportional to the number of SSNVS. SSNVs and SCNAs that were significantly observed in cCRCC TCGA data (Additional file 2: Figure S2C) are denoted. (PDF $393 \mathrm{~kb}$ )

Additional file 5: Table S2. Estimated subclonal frequencies of propagated somatic mutations in the evolutionary trajectory. Annotated information corresponding to somatic single-nucleotide variants (SSNVS) was taken from Additional file 3: Table S1. Mean cellular frequencies from a post-burn-in of 50,000 chains of MCMC analysis in PyClone are shown with subclonal information. (XLSX $15.3 \mathrm{~kb}$ )

Additional file 6: Figure S4. Performance assessment of single-cell RNA-seq data. Histograms of A singlecell frequencies in the number of generated RNA-seq reads per cell, B uniquely mapped reads, C exonic regional coverage rate, and D detected number of genes per cell. Dashed red lines indicate the mean of the $x$-axis values. Using filtering criteria of $>1 \mathrm{M}$ reads per cell, $>60 \%$ uniquely mapped rate, $>35 \%$ exonic region coverage rate, and $>5000$ detected genes, two single cells were not included in subsequent analysis (for details, see Additional file 7: Table S3). (PDF $345 \mathrm{~kb}$ )

Additional file 7: Table S3. Sequencing statistics for bulk cells and single-cell RNA-seq. Mapping information (to the hg19 genome reference) was obtained from log files after running STAR. RNA-SeQC was applied to generate a series of quality control metrics. (XLSX $21.4 \mathrm{~kb}$ )

Additional file 8: Figure S5. Evaluation of the averaged expression levels of genes with their variations across single-cell populations. A, B Filtered genes expressed as log2 ratio of transcripts per million (TPM) +1 were averaged and their standard deviations calculated across respective subgroups of single cells or the normal kidney cortex A, and across mixed subgroups B. The coefficient of variation (CV) is denoted and shown as the slope in the dotted gray line. Selected housekeeping genes are highlighted in red with their gene symbols. (PDF $1.45 \mathrm{mb}$ )

Additional file 9: Figure S6. Expression signatures of tumor cells compared to the normal kidney cortex. A, B Gene expressions of normal kidney cortexes were downloaded from the GTEx portal $(n=8$, Ver.3). To identify outlier values in normal distribution, Z-scores were estimated and are shown in radial and QQ-plots for gene sets of the stromal A and ccRCC B signatures. (PDF $135 \mathrm{~kb}$ )

Additional file 10: Figure S7. Transcriptomic similarity between primary tumor and PDX model. A, B Scatter plots show reciprocal similarities of global gene expression between bulk samples $A$, and between averaged single cells and bulk samples B. Black dotted line is the $x=y$ line with correlation coefficients (Pearson and Spearman $r$ ) for linear fit. The $x-y$ axes represent $\log 2$ ratio of TPM + 1. C Explanatory power (adjusted R-square) of gene expression of single cells to those of bulk cell population was estimated by multiple regression analysis with a randomly selected given number of cells with permutation $(\times 1000)$. Boxes show 25th to 75th percentile with 10th and 90th percentile whiskers. Median values within the boxes are represented as diamond symbols and connected in lines between boxes. Dotted vertical lines indicate the explanatory power with the total single cells. (PDF $548 \mathrm{~kb}$ )

Additional file 11: Figure S8. Gene set activation analysis for the epithelial epithelial-mesenchymal transition (EMT)-induced signature. A Positions of each dot and ellipse in Fig. 1d were fixed, and then each dot was colored (main panel) and indicated as drop lines (top and right pane/s) according to the estimated activation status in the EMT-induced signature. B Boxplots show overall reciprocal differences in the expression signatures across normal kidney cortex, bulk cells of each population, and single cells. Boxes show 25th to 75th percentile with 10th and 90th percentile whiskers. ${ }^{* * *} P<0.001$, two-tailed Student's $t$-test. (PDF $124 \mathrm{~kb}$ ) 
Additional file 12: Figure S9. Functionally grouped networks based on analysis of differentially expressed genes between PRCC and mRCC cells. A A volcano plot of gene expression (log2 ratio of TPM +1 ) contrasting pRCC cells $(n=46)$ versus mRCC cells $(n=36)$. The vertical dotted line indicates the cutoff of the false discovery rate (FDR $<0.01$ ), which was adjusted using the Benjamini-Hochberg correction for multiple-testing. The horizontal dotted lines indicate the cutoff for a significant fold change ( $\geq$ twofold). Colors indicate significantly upregulated genes in pRCC cells (red) or in mRCC cells (blue). B Visualization of enriched biological networks with upregulated genes in pRCC cells (top) or in mRCC cells (bottom) by ClueGO analysis with annotation of Gene Ontology. Term enrichment significance is determined using two-sided hypergeometric test with the Benjamini-Hochberg correction, and represented by node size. Genes that are shared between two gene ontology terms generate link lines. The most prominent gene ontology term for each functional group is highlighted in a larger font size with the text color identical to the relevant group. (PDF $1.63 \mathrm{mb}$ )

Additional file 13: Figure S10. Activity of druggable pathways of pRCC and mRCC cells in the PCA plot. The plots show heterogeneous activated status of targetable signaling pathways at single-cell resolution (in the scatter plot with colored dots) compared to bulk cells. Positions of each dot and ellipses were derived from the PCA analysis in Fig. 1d. Colors of dots (main panel) and drop lines (top and right panels) represent the relative activation status of targetable signaling pathways. Gene Set Variation Analysis (GSVA) scores were normalized to normal kidney tissue expression profiles (shown in the upper heatmap). (PDF $316 \mathrm{~kb}$ )

Additional file 14: Table S4. Results of drug screening for PRCC and mRCC tumors. Summarized list of drugs used in the screening and calculated nanomolar values of $\mathrm{IC}_{50}$. For repeated measures analysis, mRCC cells were tested twice. (PDF $94.2 \mathrm{~kb}$ )

Additional file 15: Figure S11. Repeated measurement of highthroughput drug screening. The variance in the repeated measures analysis for drug sensitivity was evaluated in PDX-mRCC cells with the identical panel of drugs. The linear regression line (black) fitted on measured $I C_{50}$ in duplicate is represented with $95 \%$ confidence intervals (gray) over a theoretical regression line (white diagonal). The strength of the linear regression and its statistical significance was determined by Pearson's correlation coefficient ( $r$ ) and one-way ANOVA test, respectively. (PDF $279 \mathrm{~kb}$ )

Additional file 16: Figure S12. Prediction of drug sensitivity across single-cell populations. A Drug sensitivity was predicted by the ridge regression model using a training set of publicly available cancer cell line expression data with measured $I C_{50}$ data for each drug. Estimated values were transformed to Z-scores across samples. Boxes show 25th to 75 th percentile with 10th and 90th percentile whiskers. Differences between groups were determined by two-tailed Student's $t$-test. ${ }^{*} P<0.05$ ${ }^{* *} P<0.01,{ }^{* *} P<0.001$. B Correlation of drug sensitivity with the relevant signaling pathways to be targeted. Uniform axis ranges were applied to all plots: $x$-axis of GSVA scores, -0.5 to 0.5 ; $y$-axis of Z-scores, -3 to 2. Linear regression was applied to estimate Pearson's correlation coefficient (r), with $95 \%$ confidence as shown in thicker light gray curves. The statistical significance of the regression was determined by one-way ANOVA test. (PDF $1.33 \mathrm{mb}$ )

Additional file 17: Figure S13. Recapitulation of poor response to pazopanib and everolimus in the patient through in vitro drug efficacy testing in 3D ECM scaffold system. In contrast to susceptibility to afatinib or dasatinib at a dose concentration of $0.5 \mu \mathrm{M}$ (Fig. 3d), we did not detect significant anticancer activity of pazopanib or everolimus, even at higher dose concentrations. Results are presented as mean values \pm SEM. (PDF $61.1 \mathrm{~kb}$ )

Additional file 18: Figure S14. Correlation of drug sensitivity with the targeted pathways in classified subpopulations. The classified single-cell subpopulations shown in Fig. 4 were evaluated for correlation between drug sensitivity and the relevant signaling pathways to be targeted. Uniform axis ranges were applied to all plots: $x$-axis of GSVA scores, -0.5 to $0.5 ; y$-axis of Z-scores, -3 to 2 . Linear regression was applied to estimate Pearson's correlation coefficient $(r)$, with $95 \%$ confidence as shown in thicker light gray curves. The statistical significance of the regression was determined by one-way ANOVA test. Although the absolute correlation coefficient ( $r$ ) was mostly high across all comparisons, some showed an insignificant correlation due to the small size of samples involved in the statistical test, compared to the overall significant correlation in unclassified single-cell populations (Fig. 3). (PDF 878 kb)

\section{Competing interests}

The authors declare that the research was conducted in the absence of any commercial or financial relationships that could be construed as a potential conflict of interest.

\section{Authors' contributions}

K-TK, HWL, D-HN, KMJ, and W-YP conceived the project; HWL, D-HN, $\mathrm{BCJ}$, and $\mathrm{KMJ}$ obtained surgical tissues and established xenograft models; $\mathrm{K}$-TK and $\mathrm{H}-\mathrm{OL}$ performed single-cell experiments and computational analysis; HWL, HJS, DEJ, SS, HK, and YS designed and performed in vitro and in vivo experiments; K-TK, HWL, DGK, KMJ, and W-YP interpreted data; $\mathrm{K}-\mathrm{TK}, \mathrm{HWL}, \mathrm{KMJ}$, and $\mathrm{W}-\mathrm{YP}$ wrote the manuscript. All authors contributed edits and approved the final manuscript.

\section{Acknowledgements}

We thank research fellows in the Samsung Genome Institute for experimental assistance and valuable comments on our work. This research was supported by the Samsung Medical Center and Health Technology R\&D Project, Ministry of Health \& Welfare (HI13C2096000013).

\section{Author details}

${ }^{1}$ Samsung Genome Institute, Samsung Medical Center, Seoul, South Korea. ${ }^{2}$ Institute for Future Medicine, Samsung Medical Center, Seoul, South Korea. ${ }^{3}$ Departments of Molecular Cell Biology, Sungkyunkwan University School of Medicine, Seoul, South Korea. ${ }^{4}$ Departments of Anatomy and Cell Biology, Sungkyunkwan University School of Medicine, Seoul, South Korea. ${ }^{5}$ Department of Health Sciences and Technology, Samsung Advanced Institute for Health Sciences \& Technology, Sungkyunkwan University, Seoul, South Korea. ${ }^{6}$ School of Mechanical Engineering, Korea University, Seoul, South Korea. ${ }^{7}$ Departments of Neurosurgery, Samsung Medical Center, Sungkyunkwan University School of Medicine, Seoul, South Korea. ${ }^{8}$ Departments of Urology, Samsung Medical Center, Sungkyunkwan University School of Medicine, Seoul, South Korea. ${ }^{9}$ Departments of Pharmacology and Cancer Biology, Duke University Medical Center, Durham, NC, USA. ${ }^{10}$ Departments of Radiation Oncology, Duke University Medical Center, Durham, NC, USA.

Received: 21 December 2015 Accepted: 11 April 2016 Published online: 29 April 2016

\section{References}

1. Rini $\mathrm{Bl}$, Atkins MB. Resistance to targeted therapy in renal-cell carcinoma. Lancet Oncol. 2009;10:992-1000.

2. Linehan WM, Srinivasan R, Schmidt LS. The genetic basis of kidney cancer: a metabolic disease. Nat Rev Urol. 2010;7:277-85.

3. Ravaud A, Hawkins R, Gardner JP, von der Maase H, Zantl N, Harper P, Rolland F, Audhuy B, Machiels JP, Petavy F, et al. Lapatinib versus hormone therapy in patients with advanced renal cell carcinoma: a randomized phase III clinical trial. J Clin Oncol. 2008;26:2285-91.

4. Rini BI, Escudier B, Tomczak P, Kaprin A, Szczylik C, Hutson TE, Michaelson MD, Gorbunova VA, Gore ME, Rusakov IG, et al. Comparative effectiveness of axitinib versus sorafenib in advanced renal cell carcinoma (AXIS): a randomised phase 3 trial. Lancet. 2011;378:1931-9.

5. Hudes G, Carducci M, Tomczak P, Dutcher J, Figlin R, Kapoor A, Staroslawska E, Sosman J, McDermott D, Bodrogi I, et al. Temsirolimus, interferon alfa, or both for advanced renal-cell carcinoma. N Engl J Med. 2007;356:2271-81.

6. Gupta K, Miller JD, Li JZ, Russell MW, Charbonneau C. Epidemiologic and socioeconomic burden of metastatic renal cell carcinoma (mRCC): a literature review. Cancer Treat Rev. 2008;34:193-205.

7. Gerlinger M, Rowan AJ, Horswell S, Larkin J, Endesfelder D, Gronroos E, Martinez P, Matthews N, Stewart A, Tarpey P, et al. Intratumor heterogeneity and branched evolution revealed by multiregion sequencing. $N$ Engl J Med. 2012;366:883-92. 
8. Gerlinger M, Horswell S, Larkin J, Rowan AJ, Salm MP, Varela I, Fisher R, McGranahan N, Matthews N, Santos CR, et al. Genomic architecture and evolution of clear cell renal cell carcinomas defined by multiregion sequencing. Nat Genet. 2014;46:225-33.

9. Kosari F, Parker AS, Kube DM, Lohse CM, Leibovich BC, Blute ML, Cheville JC, Vasmatzis G. Clear cell renal cell carcinoma: gene expression analyses identify a potential signature for tumor aggressiveness. Clin Cancer Res. 2005;11:5128-39.

10. Ozcan A, de la Roza G, Ro JY, Shen SS, Truong LD. PAX2 and PAX8 expression in primary and metastatic renal tumors: a comprehensive comparison. Arch Pathol Lab Med. 2012;136:1541-51.

11. Wyler L, Napoli CU, Ingold B, Sulser T, Heikenwalder M, Schraml P, Moch H. Brain metastasis in renal cancer patients: metastatic pattern, tumourassociated macrophages and chemokine/chemoreceptor expression. $\mathrm{Br} J$ Cancer. 2014;110:686-94.

12. Burrell RA, McGranahan N, Bartek J, Swanton C. The causes and consequences of genetic heterogeneity in cancer evolution. Nature. 2013;501:338-45.

13. Higashiyama M, Okami J, Maeda J, Tokunaga T, Fujiwara A, Kodama K, Imamura F, Kobayashi $\mathrm{H}$. Differences in chemosensitivity between primary and paired metastatic lung cancer tissues: In vitro analysis based on the collagen gel droplet embedded culture drug test (CD-DST). J Thorac Dis. 2012:4:40-7.

14. Xu X, Hou Y, Yin X, Bao L, Tang A, Song L, Li F, Tsang S, Wu K, Wu H, et al. Single-cell exome sequencing reveals single-nucleotide mutation characteristics of a kidney tumor. Cell. 2012;148:886-95.

15. Dancey JE, Bedard PL, Onetto N, Hudson TJ. The genetic basis for cancer treatment decisions. Cell. 2012;148:409-20.

16. Cohen AA, Geva-Zatorsky N, Eden E, Frenkel-Morgenstern M, Issaeva I, Sigal A, Milo R, Cohen-Saidon C, Liron Y, Kam Z, et al. Dynamic proteomics of individual cancer cells in response to a drug. Science. 2008;322:1511-6.

17. Spencer SL, Gaudet S, Albeck JG, Burke JM, Sorger PK. Non-genetic origins of cell-to-cell variability in TRAlL-induced apoptosis. Nature. 2009;459:428-32.

18. Costello JC, Heiser LM, Georgii E, Gonen M, Menden MP, Wang NJ, Bansal M, Ammadud-din M, Hintsanen P, Khan SA, et al et al. A community effort to assess and improve drug sensitivity prediction algorithms. Nat Biotechnol. 2014;32:1202-12.

19. Klijn C, Durinck S, Stawiski EW, Haverty PM, Jiang Z, Liu H, Degenhardt J, Mayba O, Gnad F, Liu J, et al. A comprehensive transcriptional portrait of human cancer cell lines. Nat Biotechnol. 2015;33:306-12.

20. Julien S, Merino-Trigo A, Lacroix L, Pocard M, Goere D, Mariani P, Landron S, Bigot L, Nemati F, Dartigues $P$, et al. Characterization of a large panel of patient-derived tumor xenografts representing the clinical heterogeneity of human colorectal cancer. Clin Cancer Res. 2012;18:5314-28.

21. Ding L, Ellis MJ, Li S, Larson DE, Chen K, Wallis JW, Harris CC, McLellan MD, Fulton RS, Fulton LL, et al. Genome remodelling in a basal-like breast cancer metastasis and xenograft. Nature. 2010;464:999-1005.

22. DeRose YS, Wang G, Lin YC, Bernard PS, Buys SS, Ebbert MT, Factor R, Matsen C, Milash BA, Nelson E, et al. Tumor grafts derived from women with breast cancer authentically reflect tumor pathology, growth, metastasis and disease outcomes. Nat Med. 2011;17:1514-20.

23. Kreso A, O'Brien CA, van Galen P, Gan Ol, Notta F, Brown AM, Ng K, Ma J, Wienholds E, Dunant C, et al. Variable clonal repopulation dynamics influence chemotherapy response in colorectal cancer. Science. 2013;339:543-8.

24. Kim KT, Lee HW, Lee HO, Kim SC, Seo YJ, Chung W, Eum HH, Nam DH, Kim J, Joo KM, Park WY. Single-cell mRNA sequencing identifies subclonal heterogeneity in anti-cancer drug responses of lung adenocarcinoma cells. Genome Biol. 2015;16:127.

25. The Cancer Genome Atlas Research Network. Comprehensive molecular characterization of clear cell renal cell carcinoma. Nature. 2013;499:43-9.

26. Erdogan F, Demirel A, Polat O. Prognostic significance of morphologic parameters in renal cell carcinoma. Int J Clin Pract. 2004:58:333-6.

27. Tsai JH, Yang J. Epithelial-mesenchymal plasticity in carcinoma metastasis. Genes Dev. 2013;27:2192-206.

28. Mikami S, Oya M, Mizuno R, Kosaka T, Katsube K, Okada Y. Invasion and metastasis of renal cell carcinoma. Med Mol Morphol. 2014;47:63-7.

29. Heng DY, Xie W, Regan MM, Harshman LC, Bjarnason GA, Vaishampayan UN, Mackenzie M, Wood L, Donskov F, Tan MH, et al. External validation and comparison with other models of the International Metastatic Renal-Cell Carcinoma Database Consortium prognostic model: a population-based study. Lancet Oncol. 2013;14:141-8.
30. Geeleher P, Cox NJ, Huang RS. Clinical drug response can be predicted using baseline gene expression levels and in vitro drug sensitivity in cell lines. Genome Biol. 2014;15:R47.

31. Garnett MJ, Edelman EJ, Heidorn SJ, Greenman CD, Dastur A, Lau KW, Greninger P, Thompson IR, Luo X, Soares J, et al. Systematic identification of genomic markers of drug sensitivity in cancer cells. Nature. 2012;483:570-5.

32. Smith K, Gunaratnam L, Morley M, Franovic A, Mekhail K, Lee S. Silencing of epidermal growth factor receptor suppresses hypoxia-inducible factor-2driven VHL-/- renal cancer. Cancer Res. 2005:65:5221-30.

33. Moch H, Sauter G, Buchholz N, Gasser TC, Bubendorf L, Waldman FM, Mihatsch MJ. Epidermal growth factor receptor expression is associated with rapid tumor cell proliferation in renal cell carcinoma. Hum Pathol. 1997:28:1255-9.

34. Stumm G, Eberwein S, Rostock-Wolf S, Stein H, Pomer S, Schlegel J, Waldherr R. Concomitant overexpression of the EGFR and erbB-2 genes in renal cell carcinoma (RCC) is correlated with dedifferentiation and metastasis. Int J Cancer. 1996:69:17-22.

35. Yonezawa $Y$, Nagashima $Y$, Sato H, Virgona N, Fukumoto K, Shirai S, Hagiwara $H$, Seki $T$, Ariga $T$, Senba $H$, et al. Contribution of the Src family of kinases to the appearance of malignant phenotypes in renal cancer cells. Mol Carcinog. 2005;43:188-97.

36. Zhao Y, Li X, Sun X, Zhang Y, Ren H. EMT phenotype is induced by increased Src kinase activity via Src-mediated caspase-8 phosphorylation. Cell Physiol Biochem. 2012;29:341-52.

37. Keating GM. Afatinib: a review of its use in the treatment of advanced non-small cell lung cancer. Drugs. 2014;74:207-21.

38. Chang Q, Jorgensen C, Pawson T, Hedley DW. Effects of dasatinib on EphA2 receptor tyrosine kinase activity and downstream signalling in pancreatic cancer. Br J Cancer. 2008;99:1074-82.

39. Montero JC, Seoane S, Ocana A, Pandiella A. Inhibition of SRC family kinases and receptor tyrosine kinases by dasatinib: possible combinations in solid tumors. Clin Cancer Res. 2011;17:5546-52.

40. Friedrich J, Seidel C, Ebner R, Kunz-Schughart LA. Spheroid-based drug screen: considerations and practical approach. Nat Protoc. 2009;4:309-24.

41. Shin Y, Han S, Jeon JS, Yamamoto K, Zervantonakis IK, Sudo R, Kamm RD, Chung S. Microfluidic assay for simultaneous culture of multiple cell types on surfaces or within hydrogels. Nat Protoc. 2012;7:1247-59.

42. Navin NE. Cancer genomics: one cell at a time. Genome Biol. 2014;15:452.

43. Siolas D, Hannon GJ. Patient-derived tumor xenografts: transforming clinical samples into mouse models. Cancer Res. 2013;73:5315-9.

44. Wu X, Northcott PA, Dubuc A, Dupuy AJ, Shih DJ, Witt H, Croul S, Bouffet E, Fults DW, Eberhart CG, et al. Clonal selection drives genetic divergence of metastatic medulloblastoma. Nature. 2012;482:529-33.

45. Klein CA. Parallel progression of primary tumours and metastases. Nat Rev Cancer. 2009:9:302-12.

46. Yokota J, Kohno T. Molecular footprints of human lung cancer progression. Cancer Sci. 2004:95:197-204.

47. Patel AP, Tirosh I, Trombetta JJ, Shalek AK, Gillespie SM, Wakimoto H, Cahill DP, Nahed BV, Curry WT, Martuza RL, et al. Single-cell RNA-seq highlights intratumoral heterogeneity in primary glioblastoma. Science. 2014;344:1396-401.

48. Lee MC, Lopez-Diaz FJ, Khan SY, Tariq MA, Dayn Y, Vaske CJ, Radenbaugh AJ, Kim HJ, Emerson BM, Pourmand N. Single-cell analyses of transcriptional heterogeneity during drug tolerance transition in cancer cells by RNA sequencing. Proc Natl Acad Sci U S A. 2014;111: E4726-35.

49. Yu M, Ting DT, Stott SL, Wittner BS, Ozsolak F, Paul S, Ciciliano JC, Smas ME, Winokur D, Gilman AJ, et al. RNA sequencing of pancreatic circulating tumour cells implicates WNT signalling in metastasis. Nature. 2012;487:510-3.

50. Li H, Durbin R. Fast and accurate long-read alignment with BurrowsWheeler transform. Bioinformatics. 2010;26:589-95.

51. McKenna A, Hanna M, Banks E, Sivachenko A, Cibulskis K, Kernytsky A, Garimella K, Altshuler D, Gabriel S, Daly M, DePristo MA. The Genome Analysis Toolkit: a MapReduce framework for analyzing next-generation DNA sequencing data. Genome Res. 2010;20:1297-303.

52. Cibulskis K, Lawrence MS, Carter SL, Sivachenko A, Jaffe D, Sougnez C, Gabriel S, Meyerson M, Lander ES, Getz G. Sensitive detection of somatic point mutations in impure and heterogeneous cancer samples. Nat Biotechnol. 2013;31:213-9.

53. Robinson JT, Thorvaldsdottir H, Winckler W, Guttman M, Lander ES, Getz G, Mesirov JP. Integrative genomics viewer. Nat Biotechnol. 2011;29:24-6. 
54. Cingolani P, Platts A, le Wang L, Coon M, Nguyen T, Wang L, Land SJ, Lu X, Ruden DM. A program for annotating and predicting the effects of single nucleotide polymorphisms, SnpEff: SNPs in the genome of Drosophila melanogaster strain w1118; iso-2; iso-3. Fly (Austin). 2012;6:80-92.

55. Ritchie ME, Phipson B, Wu D, Hu Y, Law CW, Shi W, Smyth GK. limma powers differential expression analyses for RNA-sequencing and microarray studies. Nucleic Acids Res. 2015;43:e47.

56. Venkatraman ES, Olshen AB. A faster circular binary segmentation algorithm for the analysis of array CGH data. Bioinformatics. 2007;23:657-63.

57. Carter SL, Cibulskis K, Helman E, McKenna A, Shen H, Zack T, Laird PW, Onofrio RC, Winckler W, Weir BA, et al. Absolute quantification of somatic DNA alterations in human cancer. Nat Biotechnol. 2012;30:413-21.

58. Roth A, Khattra J, Yap D, Wan A, Laks E, Biele J, Ha G, Aparicio S, Bouchard-Cote A, Shah SP. PyClone: statistical inference of clonal population structure in cancer. Nat Methods. 2014;11:396-8.

59. Dobin A, Davis CA, Schlesinger F, Drenkow J, Zaleski C, Jha S, Batut P, Chaisson M, Gingeras TR. STAR: ultrafast universal RNA-seq aligner. Bioinformatics. 2013;29:15-21.

60. Li B, Dewey CN. RSEM: accurate transcript quantification from RNA-Seq data with or without a reference genome. BMC Bioinformatics. 2011;12:323.

61. Hanzelmann S, Castelo R, Guinney J. GSVA: gene set variation analysis for microarray and RNA-seq data. BMC Bioinformatics. 2013;14:7.

62. Yoshihara K, Shahmoradgoli M, Martinez E, Vegesna R, Kim H, Torres-Garcia W, Trevino V, Shen H, Laird PW, Levine DA, et al. Inferring tumour purity and stromal and immune cell admixture from expression data. Nat Commun. 2013:4:2612.

63. Jones J, Otu H, Spentzos D, Kolia S, Inan M, Beecken WD, Fellbaum C, Gu X, Joseph M, Pantuck AJ, et al. Gene signatures of progression and metastasis in renal cell cancer. Clin Cancer Res. 2005;11:5730-9.

64. Taube JH, Herschkowitz Jl, Komurov K, Zhou AY, Gupta S, Yang J, Hartwell K, Onder TT, Gupta PB, Evans KW, et al. Core epithelial-to-mesenchymal transition interactome gene-expression signature is associated with claudinlow and metaplastic breast cancer subtypes. Proc Natl Acad Sci U S A. 2010; 107:15449-54

65. Gatza ML, Lucas JE, Barry WT, Kim JW, Wang Q, Crawford MD, Datto MB, Kelley M, Mathey-Prevot B, Potti A, Nevins JR. A pathway-based classification of human breast cancer. Proc Natl Acad Sci U S A. 2010;107:6994-9.

66. Johnson WE, Li C, Rabinovic A. Adjusting batch effects in microarray expression data using empirical Bayes methods. Biostatistics. 2007:8:118-27.

67. Bindea G, Mlecnik B, Hackl H, Charoentong P, Tosolini M, Kirilovsky A, Fridman WH, Pages F, Trajanoski Z, Galon J. ClueGO: a Cytoscape plug-in to decipher functionally grouped gene ontology and pathway annotation networks. Bioinformatics. 2009;25:1091-3.

68. Shannon P, Markiel A, Ozier O, Baliga NS, Wang JT, Ramage D, Amin N, Schwikowski B, Ideker T. Cytoscape: a software environment for integrated models of biomolecular interaction networks. Genome Res. 2003;13:2498-504.

69. Lee HW, Lee JI, Lee SJ, Cho HJ, Song HJ, da Jeong E, Seo YJ, Shin S, Joung JG, Kwon YJ, et al. Patient-derived xenografts from non-small cell lung cancer brain metastases are valuable translational platforms for the development of personalized targeted therapy. Clin Cancer Res. 2015;21:1172-82.

70. Lee DW, Choi YS, Seo YJ, Lee MY, Jeon SY, Ku B, Kim S, Yi SH, Nam DH. High-throughput screening (HTS) of anticancer drug efficacy on a micropillar/microwell chip platform. Anal Chem. 2014;86:535-42.

\section{Submit your next manuscript to BioMed Central and we will help you at every step:}

- We accept pre-submission inquiries

- Our selector tool helps you to find the most relevant journal

- We provide round the clock customer support

- Convenient online submission

- Thorough peer review

- Inclusion in PubMed and all major indexing services

- Maximum visibility for your research

Submit your manuscript at www.biomedcentral.com/submit
Biomed Central 